\title{
Designing discrete predictor-based controllers for networked control systems with time-varying delays: application to a visual servo inverted pendulum system
}

\author{
Yang Deng, Vincent Léchappé, Changda Zhang, Emmanuel Moulay, Dajun Du, Franck Plestan, Member, IEEE, \\ and Qing-Long Han, Fellow, IEEE
}

\begin{abstract}
A discrete predictor-based control method is developed for a class of linear time-invariant networked control systems with a sensor-to-controller time-varying delay and a controller-to-actuator uncertain constant delay, which can be potentially applied to vision-based control systems. The control scheme is composed of a state prediction and a discrete predictorbased controller. The state prediction is used to compensate for the effect of the sensor-to-controller delay, and the system can be stabilized by the discrete predictor-based controller. Moreover, it is shown that the control scheme is also robust with respect to slight message rejections. Finally, the main theoretical results are illustrated by simulation results and experimental results based on a networked visual servo inverted pendulum system.
\end{abstract}

Index Terms-Networked control system, vision-based control, time-varying delay, inverted pendulum system, discrete predictorbased control.

\section{INTRODUCTION}

\section{A. Motivations}

$\mathbf{V}$ ISION-BASED sensors are widely used in various control applications (e.g. self-driving cars [1], [2], robots [3], UAVs [4], and inverted pendulums [5]-[7]) due to their low cost (compared with LIDAR [2, pp.176]) and the rapid progress of image-processing-based sensing techniques (e.g. indoor navigation [8], collision avoidance [9]). In such a

Manuscript received Mar 12, 2021; revised Jun 07, 2021; accepted Jul 12, 2021.

The work of Yang Deng was supported by the China Scholarship Council (CSC). The work of Dajun Du and Changda Zhang was supported in part by the National Science Foundation of China under Grant 92067106 (Corresponding author: Qing-Long Han).

Y. Deng is with the Department of Automation, Tsinghua University, Beijing, 100084, China. Email: dengyang@mail.tsinghua.edu.cn.

V. Léchappé is with the Université de Lyon, INSA de Lyon, ECL, Université Claude Bernard Lyon 1, CNRS, Ampère, F-69621, Villeurbanne, France. Email: vincent.lechappe@insa-lyon.fr.

C. Zhang and D. Du are with the Shanghai key Laboratory of Power Station Automation Technology, School of Mechatronic Engineering and Automation, Shanghai University, 200072, Shanghai, China. Emails: zhangweiran@shu.edu.cn,ddj@i.shu.edu.cn.

E. Moulay is with the XLIM (UMR CNRS 7252), Universite de Poitiers 11 bd Marie et Pierre Curie, 86073 Poitiers Cedex 9, France. Email: emmanuel.moulay@univ-poitiers.fr.

F. Plestan is with the LS2N (UMR CNRS 6004), École Centrale de Nantes, 1 Rue de la Noë, 44321 Nantes Cedex 3, France. Email: franck.plestan@ecnantes.fr.

Q.-L. Han is with the School of Science, Computing and Engineering Technologies, Swinburne University of Technology, Melbourne, Victoria 3122, Australia. Email: qhan@swin.edu.au. control system, state information can be resolved from the sampled images captured by a camera [3, pp.132] by using an image-processing algorithm. After resolution, the state information is available for the controller design, and it is transmitted to the controller through a wired or wireless sensor-to-controller communication channel that is subject to a small uncertain time-varying transmission delay of several milliseconds [10, pp.147]. After receiving state information, the controller updates the control signal and sends it to the actuator with the help of the controller-to-actuator channel. Finally, the updated control input is applied to the controlled plant. Based on the previous statements, the whole control process of such a system is subject to the following timedelays:

- the exposure time of the camera (constant);

- the computational delay introduced by the imageprocessing algorithm (long, time-varying);

- the transmission latency induced from the sensor-tocontroller communication channel (small, time-varying);

- the computation time of the control algorithm (small, constant);

- the transmission latency induced from the controller-toactuator communication channel (small, time-varying);

- the physical dead time of the actuator (constant).

Note that the time-delays introduced by data communication are time-varying because of network congestion, the communication protocol, and the real-time intensity of the communication network. The computational time of the image-processing algorithm is also time-varying since it is usually affected by the number of feature points in each frame, the number of image edges in each frame, and the real-time illumination intensity.

Based on the discussions above, the vision-based control system can be modeled as a networked control system (NCS) [11] with sensor-to-controller time-varying delay and the controller-to-actuator uncertain constant delay ${ }^{1}$. Thus, due to its high potential for application (e.g. an indoor mobile robot, or a UAV in weak GPS environment [4]), it is important to develop new control strategies to stabilize the system.

\footnotetext{
${ }^{1}$ The controller-to-actuator delay is the sum of a constant delay (dead time of the actuator) and a small time-varying delay (controller-to-actuator data transmission latency). Therefore, it can be equivalently modeled as an uncertain constant delay.
} 


\section{B. Interests of the discrete-time method}

Some discussions are given in this subsection in order to explain the reason why the discrete-time control method is adopted. The main challenges for the vision-based control systems mentioned in Subsection I-A are given in the sequel:

- Problem 1: The image-processing algorithm needs a long computational time (compared with the transmission delays). As a result, a long sensor-to-controller timevarying delay is introduced [6], [12];

- Problem 2: The vision-based control system can only run at a low sampling rate. Indeed, the sampling rate of such a system depends on the frame rate of the camera and the computational time of the image-processing algorithm. On the one hand, if the frame rate is high, then the quality of the captured images will be lower (i.e. low-contrast [5, pp.8138]), and it may introduce more measurement errors. On the other hand, a high sampling rate will make unprocessed images accumulate if no distributed computation is applied [13, pp.554], and this problem will make the control task more complicated.

After discussing the main issues of the vision-based control systems modeled as an NCS, three main control solution to NCSs [14], [15] are recalled:

- Discrete-time method [16]-[18]: This method turns the original NCS into a discrete-time extended system, where the control law is designed for the extended system in the discrete-time domain;

- Impulsive system method [19]: Using this method, an NCS is considered as a hybrid system (i.e. continuoustime system with state jumps), and the discontinuous Lyapunov functional is used for the stability analysis;

- Time-delay method [20]-[22]: This method treats the effects of sampling \& $\mathrm{ZOH}$ (zero-order hold) as a timevarying delay, where a Lyapunov-Krasovskii stability analysis is provided.

To solve Problem 1, predictor-based controllers designed by using the discrete-time method [18] and the time-delay method [23] are able to deal with long time-delays, whereas the impulsive system method is not effective.

Next, the arguments given in [15, pp.61] explain that the discrete-time method is less conservative in the maximum allowable transmission interval (MATI) for linear time-invariant (LTI) networked control systems, which indicates that this method can stabilize the LTI networked control systems with a lower sampling rate. Thus, it can better meet the requirement of Problem 2.

In conclusion, the discrete-time method can solve Problems 1 and 2 together, and is suitable for LTI vision-based control systems. Therefore, the main objective of this paper is to develop a discrete predictor-based control technique for a class of LTI networked control systems, and then implement the control algorithm on a real vision-based control system.

\section{Contributions and organizations}

This paper proposes a control solution to stabilize NCSs with sensor-to-controller time-varying delay and controllerto-actuator uncertain constant delay, which can be applied to vision-based control systems. The controller design is based on the discrete-time extended system representation [16]-[18], [24] in order to deal with long time-delays and a long sampling period. The message rejection (packet disordering) is also considered. The main theoretical contribution of this paper is threefold:

- compared with work [18], where the network-induced delay is a constant, this paper considers that the networkinduced delay is time-varying;

- in [24], discrete predictor-based control of the sampleddata system with a single uncertain constant input-delay is developed, but the explicit upper bound on the allowable delay uncertainty is not given; this bound is calculated in detail by this paper;

- the effects of the time-varying delays are analyzed in [17], [25], but they did not consider the effect of message rejection (packet disordering) caused by the time-varying delays, whereas the proposed method deals with the message rejection phenomenon in the sensor-to-controller channel.

Furthermore, the proposed control scheme is implemented on the networked inverted pendulum visual servo system (NIPVSS) introduced in [6], [7], the experimental results are given in Section VII.

In conclusion, this paper provides a control scheme for vision-based control systems (which can be modeled as a class of LTI networked control systems with time-varying delays), and the proposed method has the following main features:

- The state prediction technique is used to compensate for the effects of sensor-to-controller time-varying delay, and also deals with slight message rejection.

- The discrete predictor-based controller is designed to stabilize the LTI plant which is subject to controllerto-actuator uncertain constant delay. The benefit of this control algorithm is lower conservativeness in the sense of MATI, as stated in Subsection I-B.

- The experimental validation of the proposed control technique on the NIPVSS test bench, which illustrates that the proposed method is able and suitable to cope with the real visual servo control problem. Moreover, based on the experimental results presented in Subsection VII-C, it is observed that the proposed method can provide better control performance than the non-predictive control method.

The rest of the paper is organized as follows. The problem statement is given in Section II in order to explain modeling and some necessary assumptions. The extended system representation, the state prediction technique, and the discrete predictor-based controller are introduced in Section III. The main theoretical results for the unperturbed plant are presented in Section IV. Then the theoretical results are extended to the case with external disturbances in Section V, and they are illustrated by simulation results of Section VI and experimental results provided in Section VII. Finally, a conclusion is presented in Section VIII. 


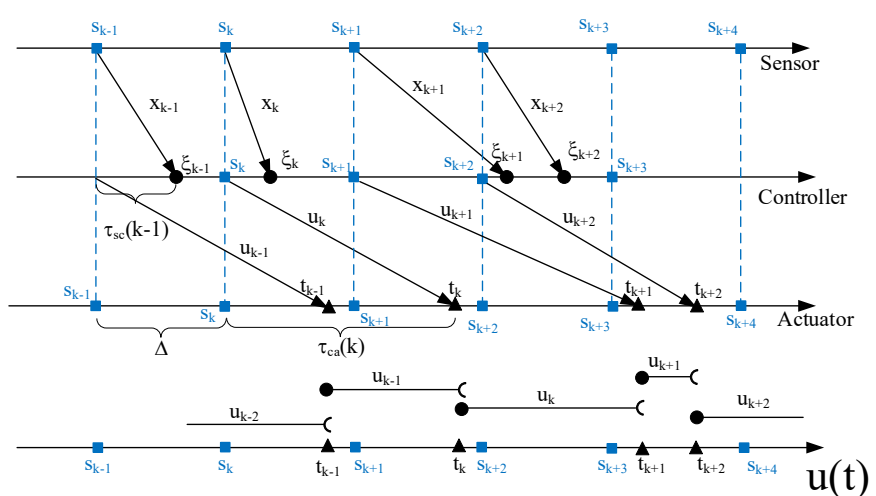

Fig. 1: Timing diagram of the control scheme with time-driven sensor, time-driven controller and event-driven actuator.

\section{Problem StATEMEnT}

\section{A. Notations}

In this paper, the following notations are used. The symbols $\mathbb{N}$ and $\mathbb{N}_{+}$represent the sets of non-negative integers and strictly positive integers. The set of all real matrices with $m$ rows and $n$ columns reads as $\mathbb{R}^{m \times n}$. The identity matrix of size $m \times m$ writes as $I_{m}$. The superscript " $T$ " denotes the matrix transposition. The symbol $\|\cdot\|$ gives the Euclidean norm of a vector and the 2-norm of a matrix, respectively. Finally, the maximum and minimum eigenvalues of a symmetric matrix read as $\lambda_{\max }(\cdot)$ and $\lambda_{\min }(\cdot)$.

\section{B. System modeling}

In this subsection, the modeling of the LTI networked control system for the LTI vision-based control system (as described in Subsection I-A) is introduced. Consider the following LTI plant

$$
\dot{x}(t)=A x(t)+B u(t), \quad t \geq 0
$$

controlled through a network with $A \in \mathbb{R}^{n \times n}$ and $B \in \mathbb{R}^{n \times m}$. The control input is piecewise-constant such that

$$
\begin{aligned}
& u(t)=u_{k}, \quad t \in\left[t_{k}, t_{k+1}\right), \quad k \in \mathbb{N} \\
& u(t)=0, \quad t<t_{0} .
\end{aligned}
$$

where $t_{k}$ is the instant that the $k$ th control input $u_{k}$ is applied to the plant. In this work, the sensor and the controller have time-driven behavior with a sampling period $\Delta>0$, which means that they update the state measurement and the control input each $\Delta$ seconds and send them out. The actuator is eventdriven, which applies the new control input to the plant as soon as it receives the updated control signal. Fig. 1 is the timing diagram of this method, and the sequence $\left\{s_{k}\right\}_{k \in \mathbb{N}}$ represents the sampling instants of the sensor and the updating instants of the controller. The sequence $\left\{\xi_{k}\right\}_{k \in \mathbb{N}}$ describes the arriving instants (at the controller) of the state measurement $x\left(s_{k}\right)$, and $\left\{t_{k}\right\}_{k \in \mathbb{N}}$ denotes the instants that a new control input $u_{k}$ is applied to the plant, as shown in (2). The state information of the controlled plant is periodically measured (with sampling period $\Delta>0$ ) and sent to the controller through the sensorto-controller network. Namely, at instants $s_{k}=k \Delta, k \in \mathbb{N}$, the sensor takes action and sends the state measurement to the controller. According to Fig. 1, one defines the sensorto-controller time-varying delay as $\tau_{s c}(k) \triangleq \xi_{k}-s_{k}$, and it is supposed to be bounded by:

$$
0<\tau_{s c}(k) \leq N_{1} \Delta
$$

with known $N_{1} \in \mathbb{N}_{+}$. Due to the discussions above and Fig. 1, the controller is time-driven, so it also updates the new control input at instants $s_{k}=k \Delta, k \in \mathbb{N}$. Therefore, the controller-toactuator delay is defined as $\tau_{c a}(k)=t_{k}-s_{k}$, and is modeled as an uncertain constant delay (based on the discussions given in Subsection I-A) such that

$$
\tau_{c a}(k)=\bar{\tau}_{c a}+\Delta \tau(k)
$$

where the nominal controller-to-actuator constant delay $\bar{\tau}_{c a}$ is known and bounded by

$$
\left(N_{2}-1\right) \Delta<\bar{\tau}_{c a} \leq N_{2} \Delta
$$

with known $N_{2} \in \mathbb{N}_{+}$, and the delay uncertainty is bounded by

$$
0<\Delta \tau(k) \leq \Delta
$$

Remark 1. According to the discussions of Subsection I-A, the delay uncertainty $\Delta \tau(k)$ of a visual feedback system arises from the time-varying data communication latency (e.g. transmission delay through the controller-to-actuator communication network), and this kind of time-delay is usually smaller than 10ms [10, pp.147]. However, the sampling period $\Delta$ of a real visual feedback system is always in the level of $10-40 \mathrm{~ms}$ (e.g. 11.5ms in [5], 12.5-25ms in [13], 40ms in [3]), which is greater than the delay uncertainty. Thus, (6) is not a strong constraint for control applications.

Fig. 1 shows that the controller is time-driven, but not event-driven. The main benefit of the time-driven controller is the compensation of the sensor-to-controller time-varying delay with the state prediction technique provided in Section III, without solving the Belleman equation [17] nor checking large numbers of LMIs (linear matrix inequalities) [16].

Assumption 1. Define $\bar{A}=e^{A \Delta}, \quad \bar{B}_{1}=\int_{0}^{N_{2} \Delta-\bar{\tau}_{c a}} e^{A \theta} \mathrm{d} \theta B$ and $\bar{B}_{2}=\int_{N_{2} \Delta-\bar{\tau}_{c a}}^{\Delta} e^{A \theta} \mathrm{d} \theta B$. It is assumed that the pair $\left(\bar{A}, \bar{A}^{-N_{2}+1} \bar{B}_{1}+\bar{A}^{-N_{2}} \bar{B}_{2}\right)$ is controllable.

Assumption 1 is a sufficient condition for controller design based on the discrete-time method, and some similar assumptions are given in [18], [26]. In this assumption, the nominal controller-to-actuator delay is utilized since the controller design method requires the nominal delay value [24]. The effect of the delay uncertainty is analyzed in Subsection III-B.

Next, it is assumed that the sensor and the controller are synchronized so that $\tau_{s c}(k)$ can be measured when the controller receives a new state measurement, this assumption is also made in [22], [23], [27]. Note that clock synchronization can be achieved in practice by using the time-stamp technique based on the IEEE 1588 protocol [28]. 
Remark 2. The Zeno phenomenon never occurs since the updates of the state measurement and the control law are periodic.

In [22], it is assumed that the older control inputs and the older state measurements cannot arrive at the destination later than a newer one. In other words, the sequences $\left\{t_{k}\right\}_{k \in \mathbb{N}}$ and $\left\{\xi_{k}\right\}_{k \in \mathbb{N}}$ are strictly increasing:

$$
\begin{aligned}
& t_{0}<t_{1}<\cdots<t_{k}<t_{k+1}<\cdots \\
& \xi_{0}<\xi_{1}<\cdots<\xi_{k}<\xi_{k+1}<\cdots
\end{aligned}
$$

in order to avoid message rejection (message disordering). In this paper, only a weaker assumption is required as below.

Assumption 2. The sequence $\left\{t_{k}\right\}_{k \in \mathbb{N}}$ is supposed to be strictly increasing.

Assumption 2 can also be ensured by (4), (5), and (6) due to the fact that $\tau_{c a}(k-1)<\tau_{c a}(k)+\Delta$ is always ensured by these inequalities. This assumption shows that the proposed method deals with slight message rejection in the sensor-to-controller channel.

\section{EXTENDED SYSTEM REPRESENTATION AND DISCRETE PREDICTOR-BASED CONTROLLER}

In this section, the extended system representation and the discrete predictor-based controller are introduced. Subsection III-A presents the control scheme with a controller-toactuator constant delay. Subsection III-B considers the case when the controller-to-actuator delay is an uncertain constant.

\section{A. Controller-to-actuator constant delay case}

This subsection introduces the discrete predictor-based controller [16], [18]. Firstly, one considers the simple case, i.e. there is no delay uncertainty in the controller-to-actuator channel, and the controller-to-actuator delay is constant, i.e. $\tau_{c a}(k)=\bar{\tau}_{c a}, \Delta \tau(k)=0$. Therefore, the state-translation equation [29, Chapter 2.3] between $x\left(s_{k}\right)$ and $x\left(s_{k+1}\right)$ reads as

$$
\begin{aligned}
x\left(s_{k+1}\right)= & e^{A \Delta} x\left(s_{k}\right)+\int_{s_{k}}^{t_{k-N_{2}+1}} e^{A\left(s_{k+1}-\theta\right)} \mathrm{d} \theta B u_{k-N_{2}} \\
& +\int_{t_{k-N_{2}+1}}^{s_{k+1}} e^{A\left(s_{k+1}-\theta\right)} \mathrm{d} \theta B u_{k-N_{2}+1}
\end{aligned}
$$

with $N_{2}$ defined in (5). Under Assumption 1, (8) can be equally considered as the following discrete-time system:

$$
x\left(s_{k+1}\right)=\bar{A} x\left(s_{k}\right)+\bar{B}_{1} u_{k-N_{2}+1}+\bar{B}_{2} u_{k-N_{2}} .
$$

Assumption 1 ensures that the discrete-time system (9) is controllable [26, Theorem 1]. Define the extended state $z_{k}=$
$\left[\begin{array}{lllllll}x^{T}\left(s_{k}\right) & u_{k}^{T} & \cdots & u_{k-N_{2}+1}^{T} & u_{k-N_{2}}^{T} & \cdots & u_{k-N_{1}-N_{2}-1}^{T}\end{array}\right]^{T}$.

Using (9), it leads to

$$
z_{k+1}=\underbrace{\left[\begin{array}{ccccccc}
\bar{A} & 0 & \cdots & \bar{B}_{1} & \bar{B}_{2} & \cdots & 0 \\
0 & 0 & \cdots & 0 & 0 & \cdots & 0 \\
0 & I_{m} & \cdots & 0 & 0 & \cdots & 0 \\
\vdots & \ddots & \ddots & \ddots & \ddots & \ddots & \vdots \\
0 & 0 & \cdots & I_{m} & 0 & \cdots & 0 \\
\vdots & \ddots & \ddots & \ddots & \ddots & \ddots & \vdots \\
0 & 0 & \cdots & \cdots & 0 & I_{m} & 0
\end{array}\right]}_{\mathscr{A}} z_{k}+\underbrace{\left[\begin{array}{c}
0 \\
I_{m} \\
0 \\
\vdots \\
\vdots \\
\vdots \\
0
\end{array}\right]}_{\mathscr{B}} u_{k+1} \cdot
$$

The controllability of (9) and the controllability of (10) are equivalent since (9) and (10) are two different descriptions of the same system, see [18, Remark 5]. Therefore, the controllability of the extended system (10) can be guaranteed if Assumption 1 is satisfied. It is the possible to find a statefeedback control law

$$
u_{k+1}=\mathscr{K} z_{k}
$$

that makes the matrix $\mathscr{A}+\mathscr{B} \mathscr{K}$ Schur, i.e. the closed-loop poles of $\mathscr{A}+\mathscr{B} \mathscr{K}$ are all within the unit circle of the complex plane. Thanks to the property of the Schur matrix, for all $\varepsilon>$ 0 , there exists a symmetric positive definite matrix $P$ with appropriate dimensions such that

$$
(\mathscr{A}+\mathscr{B} \mathscr{K})^{T} P(\mathscr{A}+\mathscr{B} \mathscr{K})-P=-\varepsilon I .
$$

Moreover, by applying the recursive analysis given in [31, pp.51-52], the existence and uniqueness of the solution to the continuous-time plant (1) under the digital feedback control law (11) can be ensured. To calculate the control law (11) at instant $t=s_{k+1}$, one needs the knowledge of $x\left(s_{k}\right)$ which may not arrive at the controller before $t=s_{k+1}$. To overcome this problem, one can use the latest available state measurement $x\left(s_{k-d}\right)$ (with $\left.1 \leq d \leq N_{1}\right)$ and the control history to predict the state $x\left(s_{k}\right)$ by iterating (9) for $d$ times:

$$
\left\{\begin{array}{l}
\hat{x}\left(s_{k-d+1}\right)=\bar{A} x\left(s_{k-d}\right)+\bar{B}_{1} u_{k-N_{2}-d+1}+\bar{B}_{2} u_{k-N_{2}-d}, \\
\hat{x}\left(s_{k-d+2}\right)=\bar{A} \hat{x}\left(s_{k-d+1}\right)+\bar{B}_{1} u_{k-N_{2}-d+2}+\bar{B}_{2} u_{k-N_{2}-d+1}, \\
\quad \vdots \\
\quad \vdots \\
\hat{x}\left(s_{k-1}\right)=\bar{A} \hat{x}\left(s_{k-2}\right)+\bar{B}_{1} u_{k-N_{2}-1}+\bar{B}_{2} u_{k-N_{2}-2}, \\
\hat{x}\left(s_{k}\right)=\bar{A} \hat{x}\left(s_{k-1}\right)+\bar{B}_{1} u_{k-N_{2}}+\bar{B}_{2} u_{k-N_{2}-1} .
\end{array}\right.
$$

As stated after Assumption 1, the value of $d$ can be determined by using the clock synchronization technique [28] in practice. Then, the equations above can provide the state estimation $\hat{x}\left(s_{k}\right)$ as follows:

$$
\begin{aligned}
\hat{x}\left(s_{k}\right)= & \bar{A}^{d} x\left(s_{k-d}\right)+\bar{A}^{d-1} \bar{B}_{2} u_{k-N_{2}-d}+\bar{B}_{1} u_{k-N_{2}} \\
& +\sum_{i=1}^{d-1}\left(\bar{A}^{i} \bar{B}_{1}+\bar{A}^{i-1} \bar{B}_{2}\right) u_{k-N_{2}-i} .
\end{aligned}
$$

Finally, the control law can be calculated by using statefeedback control (11) and state prediction (13).

Remark 3. Since the controller-to-actuator delay is assumed to be constant in this subsection, (13) is a perfect prediction 


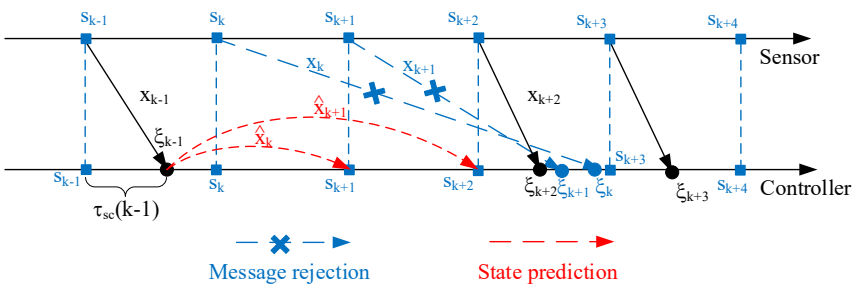

Fig. 2: Timing diagram of the sensor-to-controller channel with message rejections.

such that $\hat{x}\left(s_{k}\right)=x\left(s_{k}\right)$. If the controller-to-actuator delay is uncertain, then there exists an estimation error $e\left(s_{k}\right)=x\left(s_{k}\right)-$ $\hat{x}\left(s_{k}\right)$. The effects of the estimation error will be analyzed in Subsection III-B.

Remark 4. Consider the case when the time-delays are large (i.e. $N_{1}, N_{2}$ are large), but the sampling period $\Delta$ is small. The sizes of the matrices $\mathscr{A}, \mathscr{B}$ are large, and it may lead to numerical issues when using the Matlab pole assignment functions place and acker. In this case, one can use the alternative method provided in [18, Section 6] to compute the control law $u_{k+1}$ :

Step 1. Redefine the auxiliary state $z_{k}$ as:

$$
z_{k}=\hat{x}_{k}+\sum_{j=0}^{N_{2}-1} \bar{A}^{-j-1} \bar{B}_{1} u_{k-N_{2}+j+1}+\sum_{j=0}^{N_{2}} \bar{A}^{-j-1} \bar{B}_{2} u_{k-N_{2}+j},
$$

with $\bar{A}, \bar{B}_{1}$, and $\bar{B}_{2}$ defined in Assumption 1 .

Step 2. Define $\bar{B}=\bar{A}^{-N_{2}} \bar{B}_{1}+\bar{A}^{-N_{2}-1} \bar{B}_{2}$. Then compute the feedback gain $K_{z}$ such that the matrix $\bar{A}+\bar{B} K_{z}$ is Schur stable. Therefore, the system plant can be stabilized by using the alternative control law

$$
u_{k+1}=K_{z} z_{k} .
$$

Note that the dimension of the alternative control law (14)-(15) is equivalent to the dimension of the original system, which is smaller than the extended one. More detailed analysis about these two methods are available in [18, Remarks 8,9,10].

The state prediction technique (13) is able to deal with slight message rejection [16, pp.1576] (packet reordering [32, pp.1775]) in the sensor-to-controller network. In the communication channel, if an older data packet arrives at its destination after a newer packet, then this older one is discarded in order to ensure that the newest data is processed. This phenomenon is presented in Fig. 2, where the measurements $x\left(s_{k}\right)$ and $x\left(s_{k+1}\right)$ are neglected due to message rejection since they arrive at the controller after the measurement $x\left(s_{k+2}\right)$.

In order to deal with the message rejection shown by Fig. 2, at $t=s_{k+1}$ and $t=s_{k+2}$, one uses the technique (13) to predict the states $\hat{x}\left(s_{k}\right)$ (with the case $d=1$ ) and $\hat{x}\left(s_{k+1}\right)$ (with the case $d=2$ ), after which the control inputs $u_{k+1}$ and $u_{k+2}$ are successfully calculated even though the message rejection occurs.

Lemma 1. At least one measurement $x\left(s_{k-d}\right)$ with $1 \leq d \leq N_{1}$ is available to the controller at $t=s_{k}$.

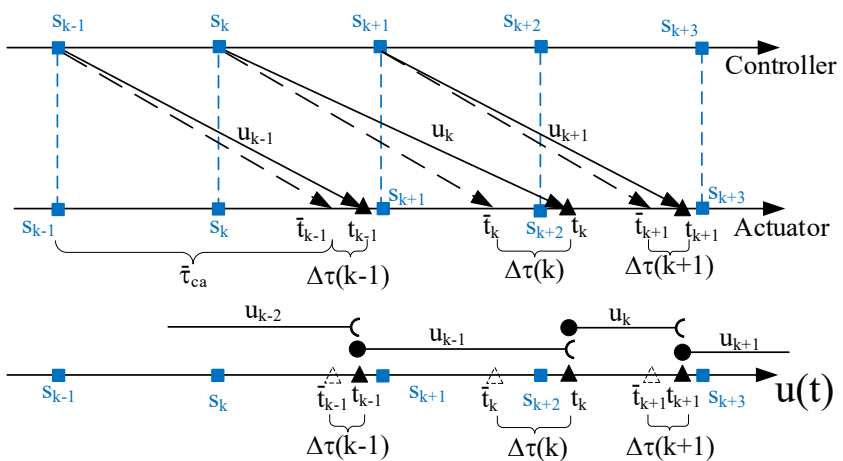

Fig. 3: Timing diagram of the controller-to-actuator channel with uncertain delays.

Proof. Firstly, because the sensor-to-controller delay is upper bounded by $N_{1} \Delta$, the measurement $x\left(s_{k-N_{1}}\right)$ must arrive at the controller before $t=s_{k}$ since

$$
\xi_{k-N_{1}}=s_{k-N_{1}}+\tau_{s c}\left(k-N_{1}\right) \leq s_{k-N_{1}}+N_{1} \Delta \leq s_{k} .
$$

Secondly, if the measurement $x\left(s_{k-N_{1}}\right)$ is not discarded, then Lemma 1 is proven with $d=N_{1}$. Thirdly, if $x\left(s_{k-N_{1}}\right)$ is rejected, then there must exist an integer $1 \leq d<N_{1}$ such that $x\left(s_{k-d}\right)$ successfully arrives at the controller and its arriving time $\xi_{k-d}$ is earlier than the arriving time of $x\left(s_{k-N_{1}}\right)$ such that

$$
\xi_{k-d} \leq \xi_{k-N_{1}} \leq s_{k} .
$$

Thus, $x\left(s_{k-d}\right)$ is available to the controller at $t=s_{k}$ and this ends the proof.

Lemma 1 ensures that the maximum value of $d$ in (13) is $N_{1}$.

\section{B. Controller-to-actuator uncertain delay case}

In this subsection, we develop the technique given in Subsection III-A, and take into account the delay uncertainty in the controller-to-actuator channel. Consider (5) and (6). The controller-to-actuator delay satisfies

$$
\left(N_{2}-1\right) \Delta<\tau_{c a}(k) \leq\left(N_{2}+1\right) \Delta .
$$

Fig. 3 shows a timing diagram of the controller-to-actuator uncertain delay case with $N_{2}=2$ for instance. Let $\left\{\bar{t}_{k}\right\}_{k \in \mathbb{N}}$ be the expected arriving times of each control input (without delay uncertainties) such that

$$
\bar{t}_{k}=s_{k}+\bar{\tau}_{c a}
$$

and the sequence $\left\{t_{k}\right\}_{k \in \mathbb{N}}$ presents the true arriving times of each control input such that

$$
t_{k}=s_{k}+\tau_{c a}(k)=\bar{t}_{k}+\Delta \tau(k) .
$$

Comparing (5) and (18), two cases must be considered.

Case 1:

$$
\left(N_{2}-1\right) \Delta<\bar{\tau}_{c a}<\tau_{c a}(k) \leq N_{2} \Delta .
$$


In this case, the delay uncertainty $\Delta \tau(k)$ is not greater than $N_{2} \Delta-\bar{\tau}_{c a}$, and it implies that

$$
s_{k}<\bar{t}_{k-N_{2}+1}<t_{k-N_{2}+1} \leq s_{k+1} .
$$

In Fig. 3, the control input $u_{k-1}$ illustrates this case. The statetranslation equation between $x\left(s_{k}\right)$ and $x\left(s_{k+1}\right)$ reads as

$$
\begin{aligned}
x\left(s_{k+1}\right)= & e^{A \Delta} x\left(s_{k}\right)+\int_{s_{k}}^{\bar{t}_{k-N_{2}+1}} e^{A\left(s_{k+1}-\theta\right)} \mathrm{d} \theta B u_{k-N_{2}} \\
& +\int_{\bar{t}_{k-N_{2}+1}}^{t_{k-N_{2}+1}} e^{A\left(s_{k+1}-\theta\right)} \mathrm{d} \theta B u_{k-N_{2}} \\
& +\int_{t_{k-N_{2}+1}}^{s_{k+1}} e^{A\left(s_{k+1}-\theta\right)} \mathrm{d} \theta B u_{k-N_{2}+1}
\end{aligned}
$$

Under Assumption 1, one has that $\bar{B}_{1}=\int_{\bar{t}_{k-N_{2}+1}}^{s_{k+1}} e^{A\left(s_{k+1}-\theta\right)} \mathrm{d} \theta B$ and $\bar{B}_{2}=\int_{s_{k}}^{\bar{t}_{k-N_{2}+1}} e^{A\left(s_{k+1}-\theta\right)} \mathrm{d} \theta B$. Let $\Delta \bar{B}_{1}\left(k-N_{2}+1\right)=$ $\int_{\bar{t}_{k-N_{2}+1}}^{t_{k-N_{2}+1}} e^{A\left(s_{k+1}-\theta\right)} \mathrm{d} \theta B$. Then (23) equals to

$$
\begin{aligned}
x\left(s_{k+1}\right)= & \bar{A} x\left(s_{k}\right)+\left[\bar{B}_{1}-\Delta \bar{B}_{1}\left(k-N_{2}+1\right)\right] u_{k-N_{2}+1} \\
& +\left[\bar{B}_{2}+\Delta \bar{B}_{1}\left(k-N_{2}+1\right)\right] u_{k-N_{2}} .
\end{aligned}
$$

Case 2:

$$
\left(N_{2}-1\right) \Delta<\bar{\tau}_{c a} \leq N_{2} \Delta<\tau_{c a}(k) .
$$

This case shows that the delay uncertainty $\Delta \tau(k)$ is greater than $N_{2} \Delta-\bar{\tau}_{c a}$, and it leads to

$$
s_{k}<\bar{t}_{k-N_{2}+1} \leq s_{k+1}<t_{k-N_{2}+1} .
$$

The control input $u_{k}$ in Fig. 3 describes this case. The statetranslation equation between $x\left(s_{k+1}\right)$ and $x\left(s_{k+2}\right)$ is given by

$$
x\left(s_{k+2}\right)=\bar{A} x\left(s_{k+1}\right)+\left[\bar{B}_{1}-\bar{B}_{1}\right] u_{k-N_{2}+2}+\left[\bar{B}_{2}+\bar{B}_{1}\right] u_{k-N_{2}+1} \text {. }
$$

Indeed, if this case occurs, the delay uncertainty $\Delta \tau(k)$ also has an influence on the system plant during the next sampling period. For instance, the control input $u_{k-1}$ of Fig. 3 is also applied to the plant on interval $\left[s_{k+2}, t_{k}\right) \subset\left[s_{k+2}, s_{k+3}\right]$ which yields

$$
\begin{aligned}
x\left(s_{k+3}\right)= & \bar{A} x\left(s_{k+2}\right)+\left[\bar{B}_{1}-\Delta \bar{B}_{1}\left(k-N_{2}+3\right)\right] u_{k-N_{2}+3} \\
& +\left[\bar{B}_{2}+\Delta \bar{B}_{1}\left(k-N_{2}+3\right)-\Delta \bar{B}_{2}\left(k-N_{2}+2\right)\right] u_{k-N_{2}+2} \\
& +\Delta \bar{B}_{2}\left(k-N_{2}+2\right) u_{k-N_{2}+1}
\end{aligned}
$$

with $\Delta \bar{B}_{2}\left(k-N_{2}+2\right)=\int_{s_{k+2}}^{t_{k}} e^{A\left(s_{k+3}-\theta\right)} \mathrm{d} \theta B$.

In conclusion, for both Case 1 and Case 2, one defines
Remark 5. If there is no delay uncertainty in the controller-toactuator channel, then the matrices $\Delta \bar{B}_{1}\left(k-N_{2}+1\right), \Delta \bar{B}_{2}(k-$ $\left.N_{2}\right)$ given in (31) are zero, and (31) is reduced to (9).

Lemma 2. If the delay uncertainty satisfies $0 \leq \Delta \tau(k) \leq \mu \leq \Delta$ for all $k \in \mathbb{N}$, then the norms of the matrices $\Delta \bar{B}_{1}(k)$ and $\Delta \bar{B}_{2}(k)$ satisfy

$$
\left\|\Delta \bar{B}_{1}(k)\right\| \leq \mu e^{\|A\| \Delta}\|B\|, \quad\left\|\Delta \bar{B}_{2}(k)\right\| \leq \mu e^{\|A\| \Delta}\|B\|
$$

for all $k \in \mathbb{N}$.

Proof. Suppose that $\Delta \tau(k)>N_{2} \Delta-\bar{\tau}_{c a}$ hold. Then one has

$$
\Delta \bar{B}_{1}(k)=\bar{B}_{1}=\int_{\bar{\tau}_{c a}-\left(N_{2}-1\right) \Delta}^{\Delta} e^{A(\Delta-\theta)} \mathrm{d} \theta B .
$$

By using the triangle inequality for integrals, (33) leads to

$$
\begin{aligned}
\left\|\Delta \bar{B}_{1}(k)\right\| & \leq \int_{\bar{\tau}_{c a}-\left(N_{2}-1\right) \Delta}^{\Delta}\left\|e^{A(\Delta-\theta)}\right\| \mathrm{d} \theta\|B\| \\
& \leq \int_{\bar{\tau}_{c a}-\left(N_{2}-1\right) \Delta}^{\Delta} e^{\|A\| \Delta} \mathrm{d} \theta\|B\| \\
& \leq\left[\Delta-\bar{\tau}_{c a}+\left(N_{2}-1\right) \Delta\right] e^{\|A\| \Delta}\|B\| \leq \mu e^{\|A\| \Delta}\|B\| .
\end{aligned}
$$

Similarly, $\left\|\Delta \bar{B}_{2}(k)\right\| \leq \mu e^{\|A\| \Delta}\|B\|$ is proven by using the same inequality (34) and the fact that $\bar{\tau}_{c a}+\Delta \tau(k)-N_{2} \Delta \leq \Delta \tau(k)$. Now, consider the case $\Delta \tau(k) \leq N_{2} \Delta-\bar{\tau}_{c a}$. One can prove that $\left\|\Delta \bar{B}_{1}(k)\right\| \leq \mu e^{\|A\| \Delta}\|B\|$ the same way as with (34), after which one has $\left\|\Delta \bar{B}_{2}(k)\right\|=0$. This ends the proof.

Similar to Subsection III-A, one uses the state prediction (13) to predict the state $x\left(s_{k}\right)$ when the expected state measurement of $x\left(s_{k}\right)$ is not available to the controller at instant $t=s_{k+1}$. However, one cannot perfectly calculate the system state $x\left(s_{k}\right)$ due to delay uncertainty $\Delta \tau(k)$, and only a state estimation $\hat{x}\left(s_{k}\right)$ can be computed. Assume that only the previous state measurement $x\left(s_{k-d}\right)$ is available to the controller at instant $t=s_{k+1}$. Then, one can calculate the state prediction error as follows.

Step 1. Firstly, the true state-translation equation (31) between $x\left(s_{k-d}\right)$ and $x\left(s_{k-d+1}\right)$ reads as

$$
\begin{aligned}
& x\left(s_{k-d+1}\right)=\bar{A} x\left(s_{k-d}\right)+\Delta \bar{B}_{2}\left(k-d-N_{2}\right) u_{k-d-N_{2}-1} \\
& \quad+\left[\bar{B}_{2}+\Delta \bar{B}_{1}\left(k-d-N_{2}+1\right)-\Delta \bar{B}_{2}\left(k-d-N_{2}\right)\right] u_{k-d-N_{2}} \\
& \quad+\left[\bar{B}_{1}-\Delta \bar{B}_{1}\left(k-d-N_{2}+1\right)\right] u_{k-d-N_{2}+1}
\end{aligned}
$$

if $\Delta \tau(k)>N_{2} \Delta-\bar{\tau}_{c a}$ and the 1-step state prediction based on $x\left(s_{k-d}\right)$ is calculated

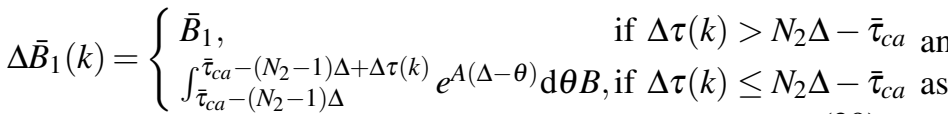

and

$$
\Delta \bar{B}_{2}(k)= \begin{cases}\int_{0}^{\bar{\tau}_{c a}+\Delta \tau(k)-N_{2} \Delta} e^{A(\Delta-\theta)} \mathrm{d} \theta B, & \text { if } \Delta \tau(k)>N_{2} \Delta-\bar{\tau}_{c a} \\ 0, & \text { if } \Delta \tau(k) \leq N_{2} \Delta-\bar{\tau}_{c a} .\end{cases}
$$

Thus, the following state-translation equation describes all the cases mentioned above:

$$
\begin{aligned}
x\left(s_{k+1}\right)= & \bar{A} x\left(s_{k}\right)+\left[\bar{B}_{1}-\Delta \bar{B}_{1}\left(k-N_{2}+1\right)\right] u_{k-N_{2}+1} \\
& +\left[\bar{B}_{2}+\Delta \bar{B}_{1}\left(k-N_{2}+1\right)-\Delta \bar{B}_{2}\left(k-N_{2}\right)\right] u_{k-N_{2}} \\
& +\Delta \bar{B}_{2}\left(k-N_{2}\right) u_{k-N_{2}-1} .
\end{aligned}
$$

$$
\hat{x}\left(s_{k-d+1}\right)=\bar{A} x\left(s_{k-d}\right)+\bar{B}_{1} u_{k-d-N_{2}+1}+\bar{B}_{2} u_{k-d-N_{2}} .
$$

Taking the difference between (35) and (36), the 1-step prediction error is

$$
\begin{aligned}
e\left(s_{k-d+1}\right)= & x\left(s_{k-d+1}\right)-\hat{x}\left(s_{k-d+1}\right) \\
= & \Delta \bar{B}_{1}\left(k-d-N_{2}+1\right)\left(u_{k-d-N_{2}}-u_{k-d-N_{2}+1}\right) \\
& +\Delta \bar{B}_{2}\left(k-d-N_{2}\right)\left(u_{k-d-N_{2}-1}-u_{k-d-N_{2}}\right) .
\end{aligned}
$$

Step 2. Secondly, one considers the estimation error $e\left(s_{k-d+2}\right)$ in a similar way, but one assumes that $x\left(s_{k-d+1}\right)$ is still not received by the controller, so the state prediction $\hat{x}\left(s_{k-d+1}\right)$ 
provided in (36) is used for the further state prediction of $x\left(s_{k-d+2}\right)$ as follows:

$$
\hat{x}\left(s_{k-d+2}\right)=\bar{A} \hat{x}\left(s_{k-d+1}\right)+\bar{B}_{1} u_{k-d-N_{2}+2}+\bar{B}_{2} u_{k-d-N_{2}+1} .
$$

Then, the true state-translation equation (31) between $x\left(s_{k-d+1}\right)$ and $x\left(s_{k-d+2}\right)$ is obtained in the sequel:

$$
\begin{aligned}
& x\left(s_{k-d+2}\right)=\bar{A} x\left(s_{k-d+1}\right)+\Delta \bar{B}_{2}\left(k-d-N_{2}+1\right) u_{k-d-N_{2}} \\
& \quad+\left[\bar{B}_{2}+\Delta \bar{B}_{1}\left(k-d-N_{2}+2\right)-\Delta \bar{B}_{2}\left(k-d-N_{2}+1\right)\right] u_{k-d-N_{2}+1} \\
& \quad+\left[\bar{B}_{1}-\Delta \bar{B}_{1}\left(k-d-N_{2}+2\right)\right] u_{k-d-N_{2}+2 .}
\end{aligned}
$$

Taking the difference between (39) and (38), one can calculate the 2-step estimation error as follows:

$$
\begin{aligned}
e\left(s_{k-d+2}\right)= & \bar{A} e\left(s_{k-d+1}\right) \\
& +\Delta \bar{B}_{1}\left(k-d-N_{2}+2\right)\left(u_{k-d-N_{2}+1}-u_{k-d-N_{2}+2}\right) \\
& +\Delta \bar{B}_{2}\left(k-d-N_{2}+1\right)\left(u_{k-d-N_{2}}-u_{k-d-N_{2}+1}\right) .
\end{aligned}
$$

Step 3. Recursively, the $d$-step state estimation error $e\left(s_{k}\right)$ based on the state measurement $x\left(s_{k-d}\right)$ reads as:

$$
\begin{aligned}
e\left(s_{k}\right)= & \bar{A}^{d-1} \Delta \bar{B}_{1}\left(k-d-N_{2}+1\right)\left(u_{k-d-N_{2}}-u_{k-d-N_{2}+1}\right) \\
& +\bar{A}^{d-1} \Delta \bar{B}_{2}\left(k-d-N_{2}\right)\left(u_{k-d-N_{2}-1}-u_{k-d-N_{2}}\right) \\
& +\bar{A}^{d-2} \Delta \bar{B}_{1}\left(k-d-N_{2}+2\right)\left(u_{k-d-N_{2}+1}-u_{k-d-N_{2}+2}\right) \\
& +\bar{A}^{d-2} \Delta \bar{B}_{2}\left(k-d-N_{2}+1\right)\left(u_{k-d-N_{2}}-u_{k-d-N_{2}+1}\right) \\
& +\cdots \\
& +\Delta \bar{B}_{1}\left(k-N_{2}\right)\left(u_{k-N_{2}-1}-u_{k-N_{2}}\right) \\
& +\Delta \bar{B}_{2}\left(k-N_{2}-1\right)\left(u_{k-N_{2}-2}-u_{k-N_{2}-1}\right) .
\end{aligned}
$$

Finally, the control law $u_{k+1}$ is calculated by

$$
u_{k+1}=\mathscr{K} \hat{z}_{k}
$$

with $\hat{z}_{k}=\left[\begin{array}{llllll}\hat{x}^{T}\left(s_{k}\right) & u_{k}^{T} & u_{k-1}^{T} & \cdots & u_{k-N_{1}-N_{2}}^{T} & u_{k-N_{1}-N_{2}-1}^{T}\end{array}\right]^{T}$.

Remark 6. Note that (42) is designed to deal with the case $1 \leq d \leq N_{1}$. If $d=0$ holds, then it implies that the state measurement $x\left(s_{k}\right)$ is available to the controller at instant $t=s_{k+1}$ and there is no need to use the state prediction (13). In this case, one can directly set $\hat{x}\left(s_{k}\right)=x\left(s_{k}\right)$, and the control law (42) is equivalent to (11).

Lemma 3. The 2-norm of a class of block matrices

$$
\Gamma=\left[\begin{array}{ccccccccc}
0 & 0 & \cdots & 0 & -B & B & 0 & \cdots & 0 \\
0 & 0 & \cdots & 0 & 0 & 0 & 0 & \cdots & 0 \\
\vdots & \vdots & \ddots & \vdots & \vdots & \vdots & \vdots & \ddots & \vdots \\
0 & 0 & \cdots & 0 & 0 & 0 & 0 & \cdots & 0
\end{array}\right]
$$

satisfies that

$$
\|\Gamma\| \leq \sqrt{2}\|B\|
$$

Proof. The 2-norm of $\Gamma$ is the induced norm [33, pp.281] such that

$$
\|\Gamma\|=\sup \{\|\Gamma x\|:\|x\|=1\}
$$

where $x$ is a vector with appropriate dimension. One assumes that $\bar{x}=\left[\begin{array}{llllll}x_{1}^{T} & \cdots & x_{j}^{T} & x_{j+1}^{T} & \cdots & x_{r}^{T}\end{array}\right]^{T}$ satisfies $\|\bar{x}\|=1$ and

$$
\|\Gamma\|=\sup \{\|\Gamma x\|:\|x\|=1\}=\|\Gamma \bar{x}\|,
$$

then one has

$$
\|\Gamma\|=\left\|\left[\begin{array}{llll}
\left(-B x_{j}+B x_{j+1}\right)^{T} & 0 & \cdots & 0
\end{array}\right]^{T}\right\|=\left\|-B x_{j}+B x_{j+1}\right\| .
$$

By using the triangle inequality, (47) is upper bounded as

$$
\|\Gamma\| \leq\|B\|\left(\left\|x_{j}\right\|+\left\|x_{j+1}\right\|\right) .
$$

Note that according to the definition of the Euclidean vector norm [33, pp.270] and the fact that $\|\bar{x}\|=\sqrt{\sum_{i=1}^{i=r}\left\|x_{i}\right\|^{2}}=1$,

$$
\left(\left\|x_{j}\right\|+\left\|x_{j+1}\right\|\right)^{2} \leq 2\left\|x_{j}\right\|^{2}+2\left\|x_{j+1}\right\|^{2} \leq 2 \sum_{i=1}^{i=r}\left\|x_{i}\right\|^{2}=2
$$

Finally, (48) and (49) imply (44), which ends the proof.

\section{MAIN THEORETICAL RESUltS}

The main theoretical results of this work are presented in this section. Before stating the main convergence result of this work, one uses the results given in Lemmas 1-3 to find the upper bound on the estimation error (41).

Lemma 4. Consider the extended states

$z_{k}=\left[\begin{array}{lllllll}x^{T}\left(s_{k}\right) & u_{k}^{T} & \cdots & u_{k-N_{2}+1}^{T} & u_{k-N_{2}}^{T} & \cdots & u_{k-N_{1}-N_{2}-1}^{T}\end{array}\right]^{T}$

and

$\hat{z}_{k}=\left[\begin{array}{lllllll}\hat{x}^{T}\left(s_{k}\right) & u_{k}^{T} & \cdots & u_{k-N_{2}+1}^{T} & u_{k-N_{2}}^{T} & \cdots & u_{k-N_{1}-N_{2}-1}^{T}\end{array}\right]^{T}$,

where there exists a constant $\Theta>0$ such that the extended error $e\left(z_{k}\right)=z_{k}-\hat{z}_{k}$ is upper bounded as follows:

$$
\left\|e\left(z_{k}\right)\right\| \leq \Theta \mu\left\|z_{k}\right\|
$$

with $\mu$ defined in Lemma 2.

Proof. Firstly, if $d=0$, then the estimation error is zero according to Remark 6, and (52) holds with an arbitrary $\Theta>0$. Then one moves on to the case $1 \leq d \leq N_{1}$, where Lemma 1 ensures that the maximum value of $d$ in (41) is $N_{1}$, and all of the control inputs mentioned in the right-hand side of (41) are contained in (50) and (51).

Secondly, the extended error between $z_{k}$ and $\hat{z}_{k}$ reads as

$$
e\left(z_{k}\right)=\left[\begin{array}{llll}
e^{T}\left(s_{k}\right) & 0^{T} & \cdots & 0^{T}
\end{array}\right]^{T} .
$$

Consider that the last term of (41) and the error term (53) yields

$$
\begin{aligned}
& {\left[\begin{array}{llll}
\Delta \bar{B}_{2}\left(u_{k-N_{2}-2}-u_{k-N_{2}-1}\right) & ]^{T} 0^{T} \quad \ldots \quad 0^{T}
\end{array}\right]^{T}=} \\
& {\left[\begin{array}{cccccccc}
0 & \cdots & 0 & -\Delta \bar{B}_{2} & \Delta \bar{B}_{2} & 0 & \cdots & 0 \\
0 & \cdots & 0 & 0 & 0 & 0 & \cdots & 0 \\
\vdots & \ddots & \vdots & \vdots & \vdots & \vdots & \ddots & \vdots \\
0 & \cdots & 0 & 0 & 0 & 0 & \cdots & 0
\end{array}\right] z_{k}}
\end{aligned}
$$


where the matrix $\Delta \bar{B}_{2}\left(k-N_{2}-1\right)$ is written as $\Delta \bar{B}_{2}$ due to space limitations. Taking the norm of (54) and applying Lemmas 2-3, it leads to

$$
\left\|\left[\begin{array}{c}
\Delta \bar{B}_{2}\left(u_{k-N_{2}-2}-u_{k-N_{2}-1}\right) \\
0 \\
\vdots \\
0
\end{array}\right]\right\|{ }^{\leq \sqrt{2} \mu e^{\|A\| \Delta}\|B\|\left\|\bar{B}_{2}\left(k-N_{2}-1\right)\right\|\left\|z_{k}\right\| .}
$$

Repeating the calculations of (54)-(55) for all of the terms presented in the right-hand side of (41), then taking the sum of them leads to

$$
\begin{aligned}
\left\|e\left(z_{k}\right)\right\| & \leq 2 \sqrt{2}\left(\sum_{i=0}^{i=d-1}\|\bar{A}\|^{i}\right) \mu e^{\|A\| \Delta}\|B\|\left\|z_{k}\right\| \\
& \leq \begin{cases}2 \sqrt{2} \frac{\|\bar{A}\|^{d}-1}{\|\bar{A}\|-1} \mu e^{\|A\| \Delta}\|B\|\left\|z_{k}\right\|, & \text { if }\|\bar{A}\| \neq 1 \\
2 \sqrt{2} d \mu e^{\|A\| \Delta}\|B\|\left\|z_{k}\right\|, & \text { if }\|\bar{A}\|=1\end{cases}
\end{aligned}
$$

Finally, by virtue of Lemma 1 , one has that $d \leq N_{1}$. Then (52) is proven with

$$
\Theta=\left\{\begin{array}{ll}
2 \sqrt{2} \frac{\|\bar{A}\| \|^{N_{1}}-1}{\|\bar{A}\|-1} e^{\|A\| \Delta}\|B\|, & \text { if }\|\bar{A}\| \neq 1 \\
2 \sqrt{2} N_{1} e^{\|A\| \Delta}\|B\|, & \text { if }\|\bar{A}\|=1
\end{array} .\right.
$$

The main convergence result of this work is given in the following theorem.

Theorem 1. Consider the fact that system (1) is controlled through a network that is subject to the sensor-to-controller time-varying delay (3) and a controller-to-actuator uncertain constant delay (4) satisfying (5)-(6). The controller is supposed to be time-driven, and the control algorithm is composed of state prediction (13) and the discrete predictor-based controller (42). If the controller-to-actuator delay uncertainty is smaller than a certain bound $\mu^{2}$ as follows:

$$
0 \leq \Delta \tau(k) \leq \mu \leq \mu^{*},
$$

for all $k \in \mathbb{N}$, then there exist constants $M>0$ and $\eta \in(0,1)$ such that the extended discrete-time system is globally exponentially stable:

$$
\left\|z_{k}\right\| \leq M\left\|z_{0}\right\| \eta^{k}
$$

and the sequence $\left\{x\left(s_{k}\right)\right\}_{k \in \mathbb{N}}$ also globally converges to zero with decay rate $\eta$.

Proof. Firstly, consider the state-translation equation (31) and the control law (42), which leads to

$$
\begin{aligned}
z_{k+1} & =\left(\mathscr{A}+\Delta \mathscr{A}_{k}\right) z_{k}+\mathscr{B} u_{k+1} \\
& =\left(\mathscr{A}+\Delta \mathscr{A}_{k}\right) z_{k}+\mathscr{B} \mathscr{K} \hat{z}_{k} \\
& =(\mathscr{A}+\mathscr{B} \mathscr{K}) z_{k}+\Delta \mathscr{A}_{k} z_{k}-\mathscr{B} \mathscr{K} e\left(z_{k}\right)
\end{aligned}
$$

${ }^{2}$ the detailed calculation of this bound is given in the proof of this theorem. with

$\Delta \mathscr{A}_{k}=\left[\begin{array}{ccccccccc}0 & \cdots & 0 & -\Delta \bar{B}_{1} & \Delta \bar{B}_{1}-\Delta \bar{B}_{2} & \Delta \bar{B}_{2} & 0 & \cdots & 0 \\ 0 & \cdots & 0 & 0 & 0 & 0 & 0 & \cdots & 0 \\ \vdots & \ddots & \vdots & \vdots & \vdots & \vdots & \vdots & \ddots & \vdots \\ 0 & \cdots & 0 & 0 & 0 & 0 & 0 & \cdots & 0\end{array}\right]$ where the indexes of $\Delta \bar{B}_{1}\left(k-N_{2}+1\right), \Delta \bar{B}_{2}\left(k-N_{2}\right)$ are omitted due to space limitations. Taking the norm of $\Delta \mathscr{A}_{k}$ and using Lemmas 2-3 gives

$$
\begin{aligned}
\left\|\Delta \mathscr{A}_{k}\right\| & \leq \sqrt{2}\left(\left\|\Delta \bar{B}_{1}\left(k-N_{2}+1\right)\right\|+\left\|\Delta \bar{B}_{2}\left(k-N_{2}\right)\right\|\right) \\
& \leq 2 \sqrt{2} e^{\|A\| \Delta}\|B\| \mu .
\end{aligned}
$$

Define the discrete-time Lyapunov function as

$$
V_{k}=z_{k}^{T} P z_{k}
$$

with $P$ satisfying (12). Taking the difference between $V_{k+1}$ and $V_{k}$ leads to

$$
\begin{aligned}
\Delta V_{k}= & V_{k+1}-V_{k} \\
= & -\varepsilon z_{k}^{T} z_{k}+z_{k}^{T} \Delta \mathscr{A}_{k}^{T} P \Delta \mathscr{A}_{k} z_{k}+e^{T}\left(z_{k}\right) \mathscr{K}^{T} \mathscr{B}^{T} P \mathscr{B} \mathscr{K} e\left(z_{k}\right) \\
& +2 z_{k}^{T}(\mathscr{A}+\mathscr{B} \mathscr{K})^{T} P \Delta \mathscr{A}_{k} z_{k}-2 z_{k}^{T} \Delta \mathscr{A}_{k}^{T} P \mathscr{B} \mathscr{K} e\left(z_{k}\right) \\
& -2 z_{k}^{T}(\mathscr{A}+\mathscr{B} \mathscr{K})^{T} P \mathscr{B} \mathscr{K} e\left(z_{k}\right) .
\end{aligned}
$$

Taking the norm of the right-hand side of (64) and using (62), (52) yields

$$
\begin{aligned}
\Delta V_{k} \leq & -\varepsilon\left\|z_{k}\right\|^{2}+8 e^{2\|A\| \Delta}\|B\|^{2}\|P\| \mu^{2}\left\|z_{k}\right\|^{2} \\
& +\|\mathscr{B} \mathscr{K}\|^{2}\|P\| \Theta^{2} \mu^{2}\left\|z_{k}\right\|^{2} \\
& +4 \sqrt{2}\|\mathscr{A}+\mathscr{B} \mathscr{K}\| e^{\|A\| \Delta}\|B\|\|P\| \mu\left\|z_{k}\right\|^{2} \\
& +4 \sqrt{2}\|\mathscr{B} \mathscr{K}\| e^{\|A\| \Delta}\|B\|\|P\| \Theta \mu^{2}\left\|z_{k}\right\|^{2} \\
& +2\|\mathscr{A}+\mathscr{B} \mathscr{K}\|\|\mathscr{B} \mathscr{K}\|\|P\| \Theta \mu\left\|z_{k}\right\|^{2} .
\end{aligned}
$$

Define

$\alpha=\left(8 e^{2\|A\| \Delta}\|B\|^{2}+\|\mathscr{B} \mathscr{K}\|^{2} \Theta^{2}+4 \sqrt{2}\|\mathscr{B} \mathscr{K}\| e^{\|A\| \Delta}\|B\| \Theta\right)\|P\|$

and

$\beta=\left(4 \sqrt{2}\|\mathscr{A}+\mathscr{B} \mathscr{K}\| e^{\|A\| \Delta}\|B\|+2\|\mathscr{A}+\mathscr{B} \mathscr{K}\|\|\mathscr{B} \mathscr{K}\| \Theta\right)\|P\|$

then $(65)$ is simplified as

$$
\Delta V_{k} \leq-\left[-\alpha \mu^{2}-\beta \mu+\varepsilon\right]\left\|z_{k}\right\|^{2} .
$$

Given a constant $0<\gamma<\min \left\{\lambda_{\max }(P), \varepsilon\right\}$, and defining

$$
\mu^{*}=\min \left\{\frac{\sqrt{\beta^{2}+4 \alpha(\varepsilon-\gamma)}-\beta}{2 \alpha}, \Delta\right\},
$$

note that if $0 \leq \mu \leq \mu^{*}$, then (69) ensures that

$$
-\alpha \mu^{2}-\beta \mu+(\varepsilon-\gamma) \geq 0
$$

and (68) further implies that

$$
V_{k+1}-V_{k}=\Delta V_{k} \leq-\gamma\left\|z_{k}\right\|^{2} \leq-\frac{\gamma}{\lambda_{\max }(P)} V_{k} .
$$


Since $0<\gamma<\lambda_{\max }(P)$, it follows that $0<1-\gamma / \lambda_{\max }(P)<1$. Finally, (71) and (50) lead to

$$
\left\|x\left(s_{k}\right)\right\| \leq\left\|z_{k}\right\| \leq \underbrace{\sqrt{\frac{\lambda_{\max }(P)}{\lambda_{\min }(P)}}}_{M}(\underbrace{\sqrt{1-\frac{\gamma}{\lambda_{\max }(P)}}}_{\eta})^{k}\left\|z_{0}\right\| .
$$

Considering the fact that $1-\gamma / \lambda_{\max }(P) \in(0,1)$, the proof of Theorem 1 is finished.

Remark 7. If $\Delta \tau(k)=0$, then Theorem 1 implies that the proposed method stabilizes NCSs with a long $\tau_{s c}(k)$, long $\Delta$ and long $\bar{\tau}_{c a}$ by virtue of the extended system representation (10) and the perfect state prediction (13). On the one hand, suppose that $\Delta \tau(k)=0$ holds. Then, the stability condition (68) is reduced to $\Delta V_{k} \leq-\varepsilon\left\|z_{k}\right\|^{2}$ which is not constrained by the sizes of $\tau_{s c}(k), \bar{\tau}_{c a}$, and $\Delta$ (given that $\mu$ is the upper bound on $\Delta \tau(k))$; on the other hand, long time-delays can be endured by the proposed method since the effects of the timedelays are converted to the structure of the delay-free extended system (10). A similar discussion about the above benefit of the discrete-time approach is also given in [18, Section 7.1].

Remark 8. In Theorem 1, the exponential convergence of the discrete-time sequence $\left\{x\left(s_{k}\right)\right\}_{k \in \mathbb{N}}$ is obtained. Indeed, since the original system (1) is linear, the continuous-time exponential convergence of $x(t)$ can be derived if the following conditions hold:

- the matrix A does not have any eigenvalue with the form of $(2 \pi / \Delta) \cdot k j$;

- if $\lambda$ is an eigenvalue of $A$ whose real part is nonnegative, then $\lambda+(2 \pi / \Delta) \cdot k j$ is not an eigenvalue of $A$;

for any nonzero integer $k$, a detailed analysis is given in [30, Section VIII].

Theorem 1 provides a discrete-time control scheme for a class of NCSs with sensor-to-controller time-varying delay, and the robustness to delay uncertainty is also analyzed.

\section{Results With EXTERnal Disturbance}

In this section, the main theoretical results of Theorem 1 are extended to a case with external disturbance, and it will be shown that the discrete-time extended system $\left\{z_{k}\right\}$ is globally ISS (input-to-state stable) even with an external disturbance. In this case, the system plant is redefined as:

$$
\dot{x}(t)=A x(t)+B u(t)+D w(t)
$$

with external disturbance $w(t) \in \mathbb{R}^{p}$ and $D \in \mathbb{R}^{n \times p}$. Therefore, the following ISS property can be obtained:

Proposition 1. Consider the perturbed system (73) controlled through the network with the sensor-to-controller time-varying delay (3) and the controller-to-actuator uncertain constant delay (4) such that conditions (5)-(6) are satisfied. Under the same conditions as Theorem 1, the discrete-time predictorbased controller composed of (13) and (42) is able to guarantee that the closed-loop system of $\left\{z_{k}\right\}$ is globally ISS.
Sketch of Proof. The sketch of proof is almost the same as the one of Theorem 1, except that the effects of the external disturbance are taken into account.

Firstly, consider the state-translation equation (31). It is modified to the following perturbed equation:

$$
\begin{aligned}
x\left(s_{k+1}\right)= & \bar{A} x\left(s_{k}\right)+\left[\bar{B}_{1}-\Delta \bar{B}_{1}\left(k-N_{2}+1\right)\right] u_{k-N_{2}+1} \\
& +\left[\bar{B}_{2}+\Delta \bar{B}_{1}\left(k-N_{2}+1\right)-\Delta \bar{B}_{2}\left(k-N_{2}\right)\right] u_{k-N_{2}} \\
& +\Delta \bar{B}_{2}\left(k-N_{2}\right) u_{k-N_{2}-1}+W_{k+1},
\end{aligned}
$$

with $W_{k+1} \triangleq \int_{s_{k}}^{s_{k+1}} e^{A\left(s_{k+1}-\theta\right)} D w(\theta) \mathrm{d} \theta$, where the disturbance term is bounded as follows:

$$
\left\|W_{k+1}\right\| \leq \Delta \cdot e^{\|A\| \Delta}\|D\| \sup _{s \in\left[s_{k}, s_{k+1}\right]}\|w(s)\| .
$$

The new state-translation equation (74) implies that the effects of the external disturbance can not only influence the current state, but also be accumulated in the state prediction procedure. Namely, applying (74) to (37), (40), and (41) yields the 1-step perturbed estimation error:

$$
\begin{aligned}
e\left(s_{k-d+1}, W\right) & =\Delta \bar{B}_{1}\left(k-d-N_{2}+1\right)\left(u_{k-d-N_{2}}-u_{k-d-N_{2}+1}\right) \\
& +\Delta \bar{B}_{2}\left(k-d-N_{2}\right)\left(u_{k-d-N_{2}-1}-u_{k-d-N_{2}}\right)+W_{k-d+1},
\end{aligned}
$$

the 2-step perturbed estimation error:

$$
\begin{aligned}
e\left(s_{k-d+2}, W\right)= & \bar{A} e\left(s_{k-d+1}, W\right)+W_{k-d+2} \\
& +\Delta \bar{B}_{1}\left(k-d-N_{2}+2\right)\left(u_{k-d-N_{2}+1}-u_{k-d-N_{2}+2}\right) \\
& +\Delta \bar{B}_{2}\left(k-d-N_{2}+1\right)\left(u_{k-d-N_{2}}-u_{k-d-N_{2}+1}\right),
\end{aligned}
$$

and the $d$-step perturbed estimation error:

$$
\begin{aligned}
e\left(s_{k}, W\right)= & \bar{A}^{d-1} \Delta \bar{B}_{1}\left(k-d-N_{2}+1\right)\left(u_{k-d-N_{2}}-u_{k-d-N_{2}+1}\right) \\
& +\bar{A}^{d-1} \Delta \bar{B}_{2}\left(k-d-N_{2}\right)\left(u_{k-d-N_{2}-1}-u_{k-d-N_{2}}\right) \\
& +\bar{A}^{d-2} \Delta \bar{B}_{1}\left(k-d-N_{2}+2\right)\left(u_{k-d-N_{2}+1}-u_{k-d-N_{2}+2}\right) \\
& +\bar{A}^{d-2} \Delta \bar{B}_{2}\left(k-d-N_{2}+1\right)\left(u_{k-d-N_{2}}-u_{k-d-N_{2}+1}\right) \\
& +\cdots \\
& +\Delta \bar{B}_{1}\left(k-N_{2}\right)\left(u_{k-N_{2}-1}-u_{k-N_{2}}\right) \\
& +\Delta \bar{B}_{2}\left(k-N_{2}-1\right)\left(u_{k-N_{2}-2}-u_{k-N_{2}-1}\right) \\
& +\sum_{i=1}^{d} \bar{A}^{d-i} W_{k-d+i} .
\end{aligned}
$$

After calculating the prediction error (78), one moves on to the stability analysis. Taking (78) into account, the main results of Lemma 4 should be modified as:

$$
\left\|e\left(z_{k}, W\right)\right\| \leq \Theta \mu\left\|z_{k}\right\|+\sum_{i=1}^{d}\|\bar{A}\|^{d-i}\left\|W_{k-d+i}\right\|
$$

Define $\bar{W}_{k+1}=\left[\begin{array}{llll}W_{k+1}^{T} & 0^{T} & \ldots & 0^{T}\end{array}\right]^{T}$. Then the extended state-translation equation (60) is recalculated as:

$$
z_{k+1}=(\mathscr{A}+\mathscr{B} \mathscr{K}) z_{k}+\Delta \mathscr{A}_{k} z_{k}-\mathscr{B} \mathscr{K} e\left(z_{k}, W\right)+\bar{W}_{k+1}
$$

where $e\left(z_{k}\right)$ takes the redefined value given in (79). Finally, considering the same Lyapunov function as given in Theorem 
1 for the discrete-time perturbed extended system (80), it leads to:

$$
\begin{aligned}
V_{k+1}-V_{k} & \leq-\gamma\left\|z_{k}\right\|^{2}+\bar{\sigma}_{1}\|\boldsymbol{w}\|^{2}+\bar{\sigma}_{2}\left\|z_{k}\right\|\|\boldsymbol{w}\| \\
& \leq-\frac{\gamma}{2}\left\|z_{k}\right\|^{2}+\left(\bar{\sigma}_{1}+\frac{\bar{\sigma}_{2}^{2}}{2 \gamma}\right)\|\boldsymbol{w}\|^{2},
\end{aligned}
$$

with $\|\boldsymbol{w}\| \triangleq \sup \left\{\|w(s)\| \mid s \in \mathbb{R}^{+}\right\}$, and

$$
\begin{gathered}
\bar{\sigma}_{1}=(\|\mathscr{B} \mathscr{K}\| \Xi+1)^{2}\|P\| e^{2\|A\| \Delta}\|D\|^{2} \cdot \Delta^{2}, \\
\bar{\sigma}_{2}=2\|P\|\left(\|\mathscr{A}+\mathscr{B} \mathscr{K}\|+2 \sqrt{2} e^{\|A\| \Delta}\|B\| \mu+\|\mathscr{B} \mathscr{K}\| \Theta \mu\right) \\
\cdot(\|\mathscr{B} \mathscr{K}\| \Xi+1) \cdot e^{\|A\| \Delta}\|D\| \cdot \Delta,
\end{gathered}
$$

where $\Xi \triangleq \sum_{i=1}^{d}\|\bar{A}\|^{d-i}$. Inequality (81) ensures that the discrete-time extended system $\left\{z_{k}\right\}$ is globally ISS under the definition given in [34, Theorem II.4] which ends the sketch of proof.

In Proposition 1, the input-to-state stability of the discretetime extended system is obtained, which implies that the proposed method can deal with the case when the system plant is perturbed by an uniformly bounded external disturbance.

\section{Simulation RESUlts}

In this section, some simulation results are provided to demonstrate the benefits of the proposed control method based on the discrete-time approach, i.e. it is able to stabilize the NCS under a low sampling rate, as described in Subsection I-B. Moreover, the message rejection is also considered in the simulation, and it is compensated by the proposed state prediction technique (13).

Consider the continuous-time unperturbed plant

$$
\dot{x}(t)=\left[\begin{array}{cc}
-1 & 0.7 \\
-0.4 & 0
\end{array}\right] x(t)+\left[\begin{array}{c}
0 \\
0.02
\end{array}\right] u(t),
$$

and the continuous-time perturbed plant

$$
\dot{x}(t)=\left[\begin{array}{cc}
-1 & 0.7 \\
-0.4 & 0
\end{array}\right] x(t)+\left[\begin{array}{c}
0 \\
0.02
\end{array}\right] u(t)+\left[\begin{array}{l}
1 \\
2
\end{array}\right] w(t),
$$

with $w(t)=0.2 \sin (2 t)$, controlled through a network. The sampling period is set to $\Delta=0.8 \mathrm{~s}$, the sensor-to-controller delay is set to

$$
\tau_{s c}(k)=\left\{\begin{array}{lll}
0.5 \mathrm{~s}, & k=4 m, & m \in \mathbb{N} \\
2.0 \mathrm{~s}, & k=4 m+1, & m \in \mathbb{N} \\
1.0 \mathrm{~s}, & k=4 m+2, & m \in \mathbb{N} \\
0.7 \mathrm{~s}, & k=4 m+3, & m \in \mathbb{N}
\end{array}\right.
$$

and the controller-to-actuator delay is chosen as an uncertain constant delay such that $1.56 \mathrm{~s} \leq \tau_{c a}(k) \leq 1.64 \mathrm{~s}$. Thus, the controller-to-actuator delay can be modeled as (4) with $\bar{\tau}_{c a}=1.56 \mathrm{~s}$ and $\Delta \tau(k) \in(0,0.08 \mathrm{~s}]$.

The time-delays of this simulation have the following features:

- the sensor-to-controller delay is upper bounded by $N_{1} \Delta$ with $N_{1}=3$, and the nominal controller-to-actuator delay $\bar{\tau}_{c a}$ satisfies (5) with $N_{2}=2$;

- the message rejection is found in the sensor-to-controller channel, and the state measurements $x\left(s_{4 m+1}\right)$ with $m \in \mathbb{N}$ are discarded;
- the controller-to-actuator delay can be greater or smaller than $N_{2} \Delta$, namely, both Cases 1 and 2 given in Subsection III-B can occur;

- the external disturbance $D w(t)$ is introduced in (84), which affects the performance of the closed-loop system. In term of the discussions above, the parameters $N_{1}=3$ and $N_{2}=2$ are determined, the extended state is then defined as

$$
\hat{z}_{k}=\left[\begin{array}{lllll}
\hat{x}^{T}\left(s_{k}\right) & u_{k}^{T} & u_{k-1}^{T} & \cdots & u_{k-6}^{T}
\end{array}\right]^{T},
$$

and the feedback controller is chosen as

$$
\begin{array}{r}
u_{k+1}=\left[\begin{array}{lllll}
0.3067 & 0.0245 & 0.7148 & -0.2276 \ldots \\
\ldots 0.0289 & -0.0016 & 0 & 0 & 0
\end{array}\right] \hat{z}_{k},
\end{array}
$$

which makes the matrix $\mathscr{A}+\mathscr{B} \mathscr{K}$ Schur. Considering the proposed parameter settings, it is possible to determine $\Theta=$ 0.3121 for Lemma 4. Next, we set $\varepsilon=1$ and solve the Lyapunov equation (12). Then, the maximum allowable upper bound on the delay uncertainty (69) under Theorem 1 (for the case of $\gamma=0.05$ ) is computed as $\mu^{*}=0.0846 \mathrm{~s}$, which is greater than the upper bounded $\mu=0.08 \mathrm{~s}$ in the simulation. Finally, the initial conditions of the simulation are set to $x(0)=\left[\begin{array}{ll}3 & -5\end{array}\right]^{T}$, and $u_{k}=0$ for all $k<0$.

In addition, considering the fact that $N_{1}=3$, one may use a state-feedback without prediction or a 1-step/ 2-step state prediction to compute control law $u_{k+1}$. The detailed control strategies for these three cases are introduced as follows:

- No state prediction is required: the control law can be directly calculated as (87) where $\hat{z}_{k}$ is replaced by $z_{k}$;

- 1-step state prediction is required: one firstly calculates $\hat{x}\left(s_{k}\right)=\bar{A} x\left(s_{k-1}\right)+\bar{B}_{1} u_{k-2}+\bar{B}_{2} u_{k-3}$, then one can use (87) to compute the control input $u_{k+1}$;

- 2-step state prediction is required: in this case, the state prediction reads as $\hat{x}\left(s_{k}\right)=\bar{A}^{2} x\left(s_{k-2}\right)+\bar{B}_{1} u_{k-2}+\left(\bar{B}_{2}+\right.$ $\left.\bar{A} \bar{B}_{1}\right) u_{k-3}+\bar{A} \bar{B}_{2} u_{k-4}$, and the estimation is then plugged into (87) for the controller design.

Namely, under the 1-step state prediction and the 2-step state prediction, the complete control laws are computed as follows:

$$
\begin{aligned}
u_{k+1}= & {\left[\begin{array}{lllll}
0.1164 & 0.1376 & 0.7148 & -0.2276 \ldots \\
\ldots 0.029 & -0.0002 & 0 & 0 & 0
\end{array}\right] z_{1-\text { step }}, } \\
u_{k+1}= & \left.\begin{array}{rrrrrr}
0.0167 & 0.1717 & 0.7148 & -0.2276 \ldots \\
\ldots 0.029 & 0 & 0.0024 & 0 & 0
\end{array}\right] z_{2-\text { step }},
\end{aligned}
$$

with

$$
\begin{aligned}
& z_{1-\text { step }}=\left[\begin{array}{lllll}
x^{T}\left(s_{k-1}\right) & u_{k}^{T} & u_{k-1}^{T} & \cdots & u_{k-6}^{T}
\end{array}\right]^{T}, \\
& z_{2-\text { step }}=\left[\begin{array}{lllll}
x^{T}\left(s_{k-2}\right) & u_{k}^{T} & u_{k-1}^{T} & \cdots & u_{k-6}^{T}
\end{array}\right]^{T} .
\end{aligned}
$$

The simulation results are provided in Fig. 4. The trajectories of the system (83) under control law (86)-(87) converge to the origin (see the blue/ red curves of Fig. 4a) for the unperturbed system (83), although the sampling period $\Delta$ is long and the sensor-to-controller delay $\tau_{s c}(k)$ is fast-varying. Next, the blue/ red dash curves of Fig. 4a illustrate that trajectories of the states converge into a small interval around the origin for the perturbed system (84). Moreover, it is observed that the message rejection that occurred in the sensorto-controller network is also successfully overcome by the control solution of Theorem 1 . 


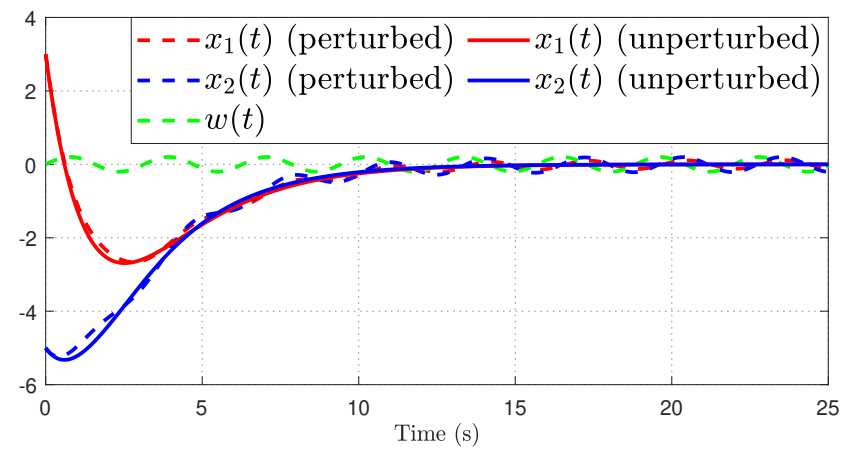

(a) State evolution of systems (83)-(84) under control law (86)-(87).

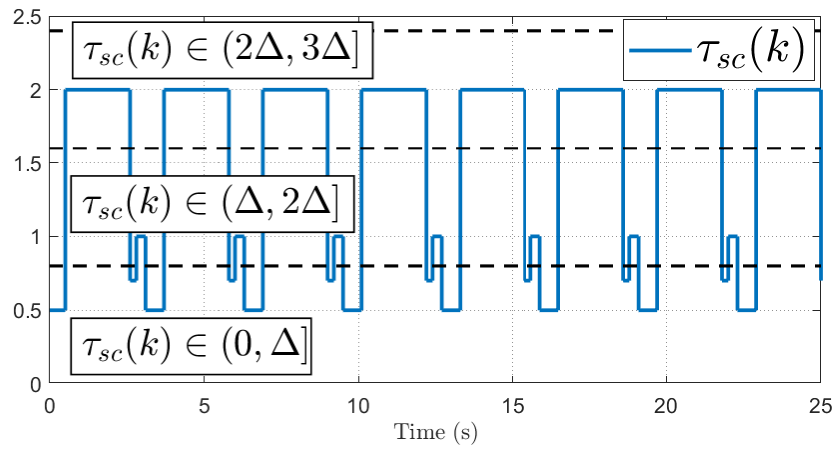

(c) Sensor-to-controller time-varying delay versus time.

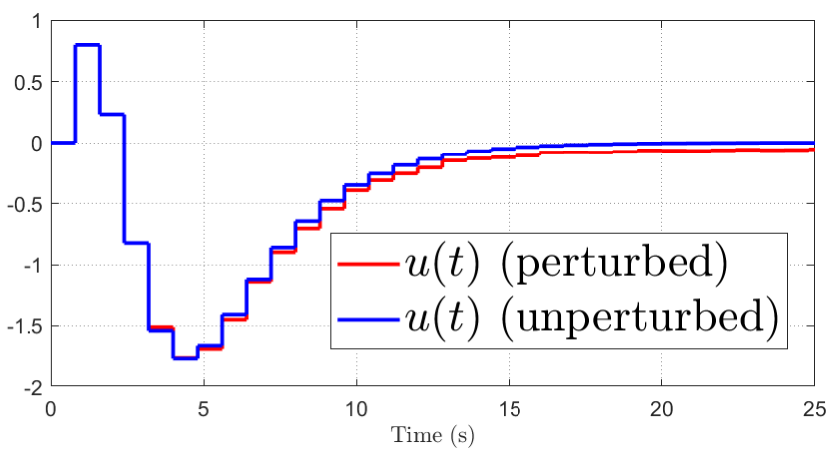

(b) Control signals (86)-(87) for two systems (83)-(84)

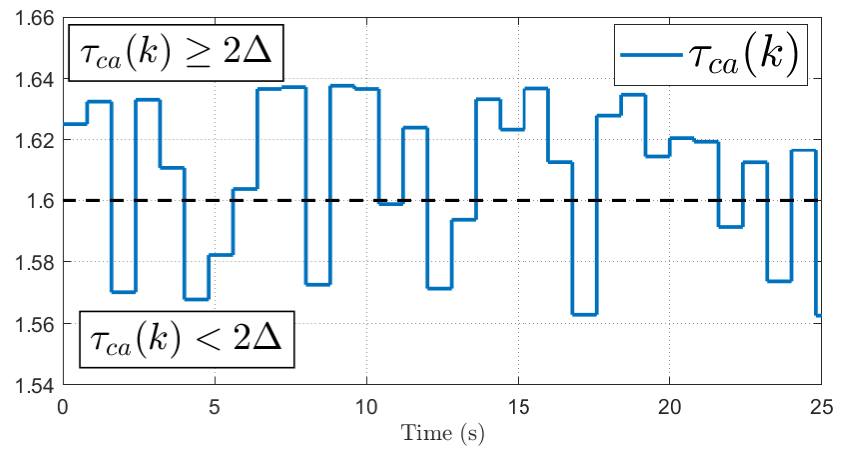

(d) Controller-to-actuator uncertain constant delay versus time.

Fig. 4: State evolution, control signal, and network-induced delays versus time for the unperturbed/ perturbed systems (83)-(84)

The simulation results presented in this section highlight the following contributions of Theorem 1 and Proposition 1:

- the state prediction technique (13) is able to deal with slight sensor-to-controller message rejections;

- due to the properties of the discrete-time approach [16], [18], the proposed method provides endurance even with a long sampling period;

- the control technique is able to cope with a uniformly bounded external disturbance, and the ISS stability/ convergence is illustrated by the simulation results.

Furthermore, the calculation of the maximum allowable delay uncertainty $\mu^{*}$ in Theorem 1 is conservative, and the choice of the feedback matrix $\mathscr{K}$ can be more flexible in practice.

\section{EXPERIMENTAL VALIDATION ON NIPVSS}

In this section, the main theoretical results of Theorem 1 are implemented on the NIPVSS test bench [6], [7] in order to test the effectiveness and the performance of the proposed control method on a real vision-based control system.

\section{A. Configuration of the NIPVSS test bench}

In this subsection, the NIPVSS test bench [6], [7] is introduced. The test bench is firstly composed of an inverted pendulum displayed in Fig. 5. The movement of the inverted pendulum is captured by an Aca640-120gm monochrome industrial camera (see Fig. 6), after which the state information $x\left(s_{k}\right)$ is obtained by the image-processing algorithm (based on Microsoft Visual Studio 2010 and OpenCV 2.4.11) on each frame. In this experimental set-up, the cart position is obtained by applying the translation between the pixel coordinate system and the world coordinate system since the camera and the moving plane are fixed. The pendulum angle is measured in the pixel coordinate system by using the edge detection technique based on the Hough Transformation [35]. Once the controller (i.e. a host computer) receives the vision-based state measurements through the communication channel, it runs the control algorithm and then sends the control signal to a $G T$ 400-SV-PCI movement control card. Finally, a MSDA023A1A servo driver receives the control signal and drives the cart to move on the rail.

\section{B. Controller design for NIPVSS based on Theorem 1}

The controller design is based on the linearized inverted pendulum plant around the straight-up position

$$
\dot{x}(t)=\underbrace{\left[\begin{array}{cccc}
0 & 0 & 1 & 0 \\
0 & 0 & 0 & 1 \\
0 & 0 & 0 & 0 \\
0 & l m g / J & 0 & 0
\end{array}\right]}_{A} x(t)+\underbrace{\left[\begin{array}{c}
0 \\
0 \\
1 \\
m l / J
\end{array}\right]}_{B} u(t)
$$

with $x(t)=\operatorname{col}\{\alpha, \theta, \dot{\alpha}, \dot{\theta}\}$. The parameters of (90) are provided in Table I. The NIPVSS is subject to the following timedelays: 


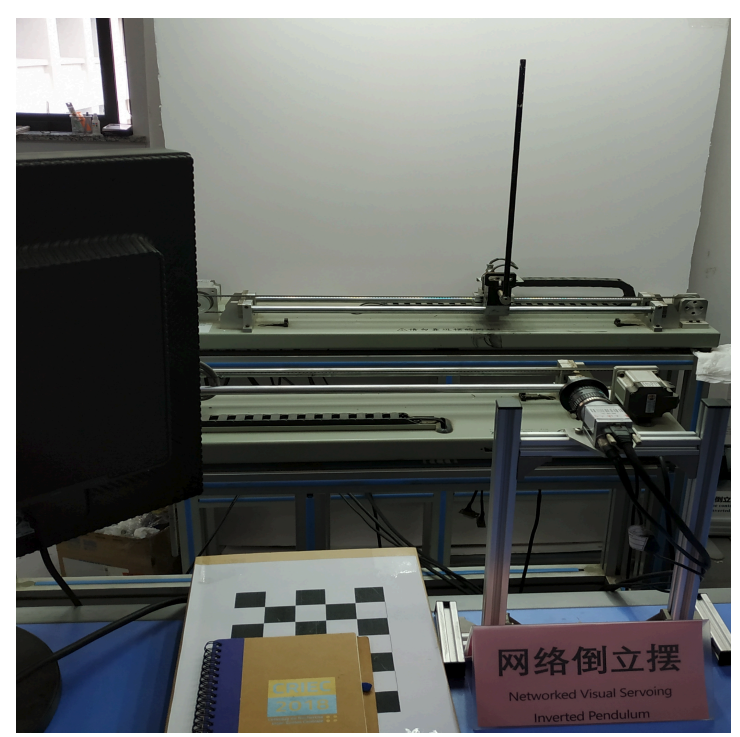

Fig. 5: The experimental set-up of the NIPVSS.

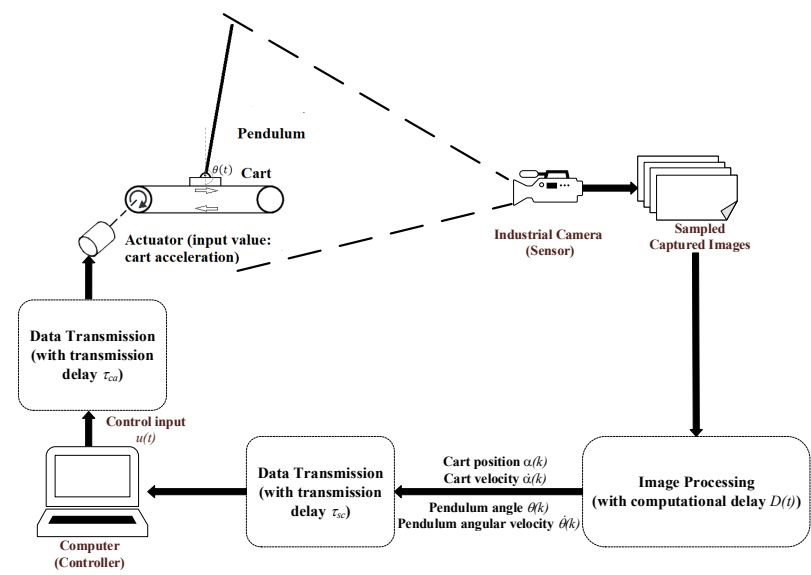

Fig. 6: Control diagram of the NIPVSS.

- the computational time $D(t) \in[0.015 \mathrm{~s}, 0.016 \mathrm{~s}]$ caused by the image-processing algorithm;

- the sensor-to-controller transmission delay bounded in $(0 \mathrm{~s}, 0.007 \mathrm{~s}]$ introduced by data communication;

- the controller-to-actuator transmission delay bounded in $(0 \mathrm{~s}, 0.007 \mathrm{~s}]$ introduced by data communication.

Thus, the control of the NIPVSS can be modeled as an NCS with the plant (90) and the time-delays mentioned above. The sensor-to-controller delay is the sum of the computational time and the transmission delay such that $\tau_{s c}(k) \in$ $(0.015 \mathrm{~s}, 0.023 \mathrm{~s}]$, and the controller-to-actuator delay satisfies $\tau_{c a}(k) \in(0 \mathrm{~s}, 0.007 \mathrm{~s}]$.

TABLE I: Model parameters of the inverted pendulum (reprint from [7, Table 1]).

\begin{tabular}{lll}
\hline$g$ & Gravitational acceleration & $9.81 \mathrm{~m} / \mathrm{s}^{2}$ \\
$m$ & Mass of the pendulum & $0.109 \mathrm{~kg}$ \\
$J$ & Moment of the inertia of the pendulum & $0.009083 \mathrm{~kg} \cdot \mathrm{m}^{2}$ \\
$l$ & Length to the pendulum center of mass & $0.25 \mathrm{~m}$ \\
$\alpha$ & Cart position & $\mathrm{m}$ \\
$\theta$ & Pendulum angle & $\mathrm{rad}$ \\
\hline
\end{tabular}

Note that the max frame rate of the industrial camera is $120 \mathrm{~Hz}$, i.e. the industrial camera can maximumly take 120 photos per second, but the image-processing algorithm cannot follow up with this sampling rate. Indeed, by considering the image-processing time $D(t)$, the image-processing algorithm cannot process more than $1 / 0.015=66.67$ frames per second. In the experiment, the sampling period is set to $\Delta=0.02 \mathrm{~s}$ in order to ensure that each frame is captured after the state information resolution of the previous frame, and a similar discussion is given in [13, pp.554].

Consider the time-delays $\tau_{s c}(k), \tau_{c a}(k)$, the sampling period $\Delta$ and the system modeling provided in (3)-(5). The parameters $N_{1}=2, \bar{\tau}_{c a}=0 \mathrm{~s}$, and $N_{2}=1$ are then determined.

Remark 9. According to Lemma 4, one should add $u_{k-i}, i=$ $\{0,1,2,3,4\}$ into the extended state (51) since $N_{1}=2$ and $N_{2}=1$. However, it is not necessary to use $u_{k-4}$ since the case described by (25) cannot occur in this experiment because

$$
\tau_{c a}(k) \leq 0.007 \mathrm{~s}<N_{2} \Delta=\Delta, \quad k \in \mathbb{N}_{+} .
$$

By virtue of (91), $u_{k-4}$ cannot influence the state-translation between $x\left(s_{k}\right)$ and $x\left(s_{k+1}\right)$, so it is not necessary to be used in the controller design.

By considering Remark 9, the following extended state is chosen to build the control law for this experiment:

$$
\hat{z}_{k}=\left[\begin{array}{lllll}
\hat{x}^{T}\left(s_{k}\right) & u_{k}^{T} & u_{k-1}^{T} & u_{k-2}^{T} & u_{k-3}^{T}
\end{array}\right]^{T} .
$$

Next, in order to guarantee the experimental control performance, the following closed-loop poles of the discrete-time extended system have been obtained by trial and error:

$$
\left\{e^{-1.6 \Delta}, e^{-1.7 \Delta}, e^{-3 \Delta}, e^{-19 \Delta}, e^{-35 \Delta}, e^{-36 \Delta}, e^{-37 \Delta}, e^{-38 \Delta}\right\},
$$

and the proposed control law reads as

$$
\begin{aligned}
& u_{k+1}=\left[\begin{array}{lllll}
0.2959 & -5.5002 & 0.5043 & -1.0435 \ldots
\end{array}\right. \\
& \left.\begin{array}{llll}
\ldots .1 .4770 & -0.9802 & 0.2888 & -0.0325
\end{array}\right] \hat{z}_{k} .
\end{aligned}
$$

Under the parameter setting of the NIPVSS, the control law $u_{k+1}$ can be calculated through a state-feedback without prediction (93) or the one with a 1-step prediction by using the same technique presented in (88)-(89), and the complete control law under the 1-step prediction is computed as:

$$
\begin{aligned}
u_{k+1}= & {\left[\begin{array}{lllll}
0.2959 & -6.1481 & 0.5103 & -1.1598 \ldots \\
& \ldots 1.4770 & -1.0361 & 0.2888 & -0.0325
\end{array}\right] z_{1-\text { step }}, }
\end{aligned}
$$

with

$$
z_{1-\text { step }}=\left[\begin{array}{lllll}
x^{T}\left(s_{k-1}\right) & u_{k}^{T} & u_{k-1}^{T} & \cdots & u_{k-6}^{T}
\end{array}\right]^{T} .
$$

In order to evaluate the control performance of the proposed method, the continuous-time LQR controller ${ }^{3}$ (with $Q=\operatorname{diag}(50,230,0,0)$ and $R=50)$ :

$$
u(t)=\left[\begin{array}{llll}
1 & -25.5765 & 1.7944 & -4.7004
\end{array}\right] x(t)
$$

provided in [37, pp.439] is used for a comparison. Note that [37] is based on the same inverted pendulum experimental set-up as Fig. 5.

\footnotetext{
${ }^{3}$ The continuous-time controller (96) is emulated [36, Section 2.2] to the sampled-data form for the experiment on the NIPVSS.
} 


\section{Experimental results}

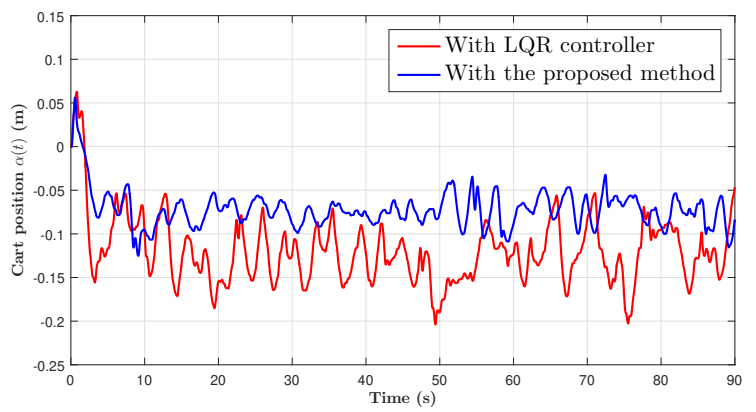

(a) Cart positions $\alpha(t)$ versus time with controllers (93) and (96).

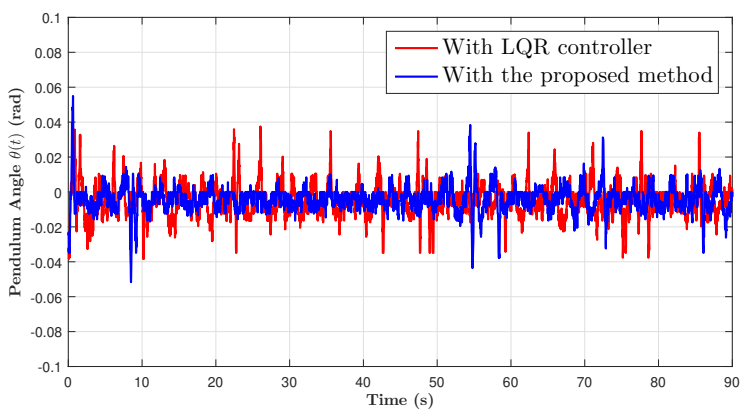

(b) Pendulum angles $\theta(t)$ versus time with controllers (93) and (96).

Fig. 7: Experimental results with the LQR controller (96) and the proposed controller (93)-(95).

The experimental results ${ }^{4}$ are presented in Fig. 7. Firstly, Fig. $7 \mathrm{~b}$ shows that the two methods can achieve good control performance on the pendulum angle $\theta(t)$ : it is maintained in a neighborhood around zero (namely, the curves are almost bounded over $[-0.04 \mathrm{rad}, 0.04 \mathrm{rad}])$. Secondly, the experimental results displayed in Fig. 7a show that the cart position $\alpha(t)$ cannot return perfectly to the original position $(\alpha(t)=0)$ due to the measurement error introduced by the image-processing algorithm [7, Remarks 3-4] and the imperfect linearized modeling of (90). However, the experimental results still confirm that the proposed method provides better control performance on the cart position than the LQR controller.

Next, as done in [7, Section V-B], the following indexes are used for the analysis of the results presented in Fig. 7:

- MCP: mean of cart position;

- SCP: standard deviation of cart position;

- MPA: mean of pendulum angle;

- SPA: standard deviation of pendulum angle.

The normalized (with respect to LQR controller (96)) performance indexes of the two controllers (93) and (96) are provided in Fig. 8: smaller the value is, better the result is. Apparently, the results obtained by the proposed controller (93) are better than the ones obtained by the LQR method (96).

\footnotetext{
${ }^{4}$ The videos of the two experiments are available at https://drive.google. com/drive/folders/1WEp7VUt5JTcUlbQf6cAt4z4ZHKKJzGjN?usp=sharing.
}

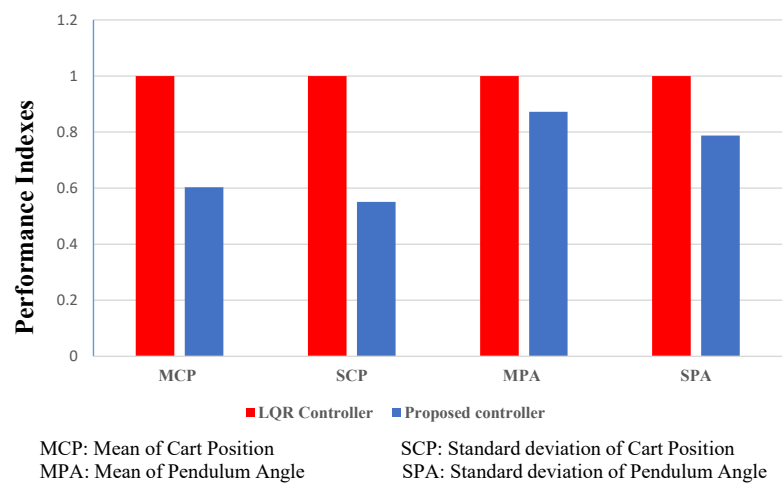

Fig. 8: Normalized (with respect to LQR controller) performance indexes of the experimental results.

The experimental results displayed by Figs. 7-8 illustrate that the proposed method is successfully implemented on the real remote visual servo control system. Moreover, the proposed method provides better experimental control performance than the non-predictive control solution (96). Furthermore, the proposed method has good control performances on such a fast motion control system, which shows that the proposed method has wide application prospects in control engineering.

\section{CONCLUSIONS}

A discrete-time control solution (state prediction and discrete predictor-based controller) is designed to stabilize a class of LTI networked control systems, and this method is applied to a networked visual servo inverted pendulum system. The state prediction technique is used to deal with the sensor-to-controller time-varying delay, and the uncertain constant controller-to-actuator delay is compensated for using the predictor-based controller. Moreover, the message rejection phenomenon is also considered in this work. The theoretical results are illustrated by the simulation and experimental results. In the future, the following research activities will be investigated:

- Theoretical part: the output-feedback control technique will be developed, some additional network-induced imperfections (e.g. quantization [18], cyber-attack [38]) will be considered, and the stochastic Lyapunov approaches will be applied in order to systematically deal with stochastic packet-dropout and message rejection at the same time. Besides, more analysis will be given in order to relax the assumption $\Delta \tau(k) \in(0, \Delta]$, and the controllerto-actuator message rejection will be studied as well.

- Experimental part: in order to improve the control performance of the cart position, the robust $H_{\infty}$ control technique will be used to calculate the feedback gain $\mathscr{K}$ in a more systematic way.

\section{REFERENCES}

[1] G. Hee Lee, F. Faundorfer, and M. Pollefeys, "Motion estimation for self-driving cars with a generalized camera," in Proc. 2013 IEEE Comput. Soc. Conf. Compu. Vision Pattern Recognit., Portland, Oregon, USA, 2013, pp. 2746-2753. 
[2] R. W. Wolcott and R. M. Eustice, "Visual localization within lidar maps for automated urban driving," in Proc. 2014 IEEE/RSJ Int. Conf. Intell. Rob. Syst, Chicago, Illinois, USA: IEEE, 2014, pp. 176-183.

[3] H. Wang, C. Vasseur, N. Christov, and V. Koncar, "Vision servoing of robot systems using piecewise continuous controllers and observers," Mech. Syst. Signal. Process., vol. 33, pp. 132-141, 2012.

[4] C. Miao and J. Li, "Autonomous landing of small unmanned aerial rotorcraft based on monocular vision in GPS-denied area," IEEE/CAA J. Autom. Sinca, vol. 2, no. 1, pp. 109-114, 2015.

[5] S. Kizir, H. Ocak, Z. Bingul, and C. Oysu, "Time delay compensated vision based stabilization control of an inverted pendulum," Int. J. Innov. Comput. Inf. Control, vol. 8, no. 12, pp. 8133-8145, 2012.

[6] G. Zhan, D. Du, and M. Fei, "Stability and stabilization for visual servo inverted pendulum system with random image processing time delay," in Proc. 43rd IECON - Annu. Conf. IEEE Ind. Electron. Soc., Beijing, China: IEEE, 2017, pp. 4325-4330.

[7] D. Du, C. Zhang, Y. Song, H. Zhou, X. Li, M. Fei, and W. Li, "Realtime $\mathrm{H}_{\infty}$ control of networked inverted pendulum visual servo systems," IEEE Trans. Cybern., 2019. vol. 50, no. 12, pp. 5113-5126, 2020.

[8] A. Garcia, E. Mattison, and K. Ghose, "High-speed vision-based autonomous indoor navigation of a quadcopter," in Proc. 2015 Int. Conf. Unmanned Aircr. Syst, ICUAS, Denver, CO, USA: IEEE, 2015, pp. 338-347.

[9] K. McGuire, G. De Croon, C. De Wagter, K. Tuyls, and H. Kappen, "Efficient optical flow and stereo vision for velocity estimation and obstacle avoidance on an autonomous pocket drone," IEEE Robot. Autom., vol. 2, no. 2, pp. 1070-1076, 2017.

[10] J. Horalek, T. Svoboda, and F. Holik, "Analysis of the wireless communication latency and its dependency on a data size," in Proc. 17th IEEE Int. Symp. Computat. Intell. Inform., Budapest, Hungary: IEEE, 2016, pp. $145-150$.

[11] X.-M. Zhang, Q.-L. Han, X. Ge, D. Ding, L. Ding, D. Yue, and C. Peng, "Networked control systems: A survey of trends and techniques," IEEE/CAA J. Autom. Sinica, vol. 7, no. 1, pp. 1-17, 2020.

[12] A. Ramirez, E. S. Espinoza, L. G. Carrillo, S. Mondié, A. García, and R. Lozano, "Stability analysis of a vision-based UAV controller: An application to autonomous road following missions," J. Intell. Rob. Syst. Theor. Appl., vol. 74, no. 1-2, pp. 69-84, 2014.

[13] H. Wu, L. Lou, C.-C. Chen, S. Hirche, and K. Kühnlenz, "Cloud-based networked visual servo control," IEEE Trans. Ind. Electron., vol. 60, no. 2, pp. 554-566, 2012

[14] X.-M. Zhang, Q.-L. Han, and X. Yu, "Survey on recent advances in networked control systems," IEEE Trans. Ind. Informat., vol. 12, no. 5, pp. 1740-1752, 2016.

[15] K. Liu, A. Selivanov, and E. Fridman, "Survey on time-delay approach to networked control," Апnи. Rev. Control, vol. 48, pp. 57-79, 2019.

[16] M. B. Cloosterman, N. Van de Wouw, W. Heemels, and H. Nijmeijer, "Stability of networked control systems with uncertain time-varying delays," IEEE Trans. Autom. Control, vol. 54, no. 7, pp. 1575-1580, 2009.

[17] S. Hu and Q. Zhu, "Stochastic optimal control and analysis of stability of networked control systems with long delay," Automatica, vol. 39, no. 11 , pp. 1877-1884, 2003

[18] V. Léchappé, E. Moulay, F. Plestan, and Q.-L. Han, "Discrete predictorbased event-triggered control of networked control systems," Automatica, vol. 107, pp. 281-288, 2019.

[19] W. Heemels, D. Nešić, A. R. Teel, and N. van de Wouw, "Networked and quantized control systems with communication delays," in Proc. 48th IEEE Conf. Decis. Control, Shanghai, China: IEEE, 2009, pp. 7929-7935.

[20] D. Yue, Q.-L. Han, and J. Lam, "Network-based robust $H_{\infty}$ control of systems with uncertainty," Automatica, vol. 41, no. 6, pp. 999-1007, 2005.

[21] D. Yue, E. Tian, and Q.-L. Han, "A delay system method for designing event-triggered controllers of networked control systems," IEEE Trans. Autom. Control, vol. 58, no. 2, pp. 475-481, 2013.

[22] A. Selivanov and E. Fridman, "Predictor-based networked control under uncertain transmission delays," Automatica, vol. 70, pp. 101-108, 2016.

[23] A. Selivanov and E. Fridman, "Observer-based input-to-state stabilization of networked control systems with large uncertain delays," Automatica, vol. 74, pp. 63-70, 2016.

[24] R. Lozano, P. Castillo, P. Garcia, and A. Dzul, "Robust prediction-based control for unstable delay systems: Application to the yaw control of a mini-helicopter," Automatica, vol. 40, no. 4, pp. 603-612, 2004

[25] Y.-J. Pan, H. Marquez, and T. Chen, "Stabilization of remote control systems with unknown time varying delays by LMI techniques," Int. J. Control, vol. 79, no. 07, pp. 752-763, 2006.
[26] C. Ionete, A. Cela, M. B. Gaid, and A. Reama, "Controllability and observability of linear discrete-time systems with network induced variable delay," IFAC Proc. Vol. (IFAC-PapersOnline), vol. 41, no. 2 , pp. 4216-4221, 2008.

[27] W. Zhang, M. S. Branicky, and S. M. Phillips, "Stability of networked control systems," IEEE Control Syst. Mag., vol. 21, no. 1, pp. 84-99, 2001.

[28] T. Cooklev, J. C. Eidson, and A. Pakdaman, "An implementation of ieee 1588 over IEEE 802.11 b for synchronization of wireless local area network nodes," IEEE Trans. Instrum. Meas., vol. 56, no. 5, pp. $1632-1639,2007$

[29] K. J. Aström and B. Wittenmark, Computer-controlled systems: theory and design third edition. Mineola, New York, NY, USA: Dover Publications, Inc., 2011.

[30] B. A. Francis and T. T. Georgiou, "Stability theory for linear timeinvariant plants with periodic digital controllers," IEEE Trans. Autom. Control, vol. 33, no. 9, pp. 820-832, 1988.

[31] I. Karafyllis, M. Malisoff, F. Mazenc, and P. Pepe, Recent results on nonlinear delay control systems. Cham, Switzerland: Springer International Publishing, 2016.

[32] J. Li, Q. Zhang, and M. Cai, "Modelling and robust stability of networked control systems with packet reordering and long delay," Int. J. Control, vol. 82, no. 10, pp. 1773-1785, 2009.

[33] C. D. Meyer, Matrix Analysis and Applied Linear Algebra. Philadelphia, PA, USA: SIAM: Society for Industrial and Applied Mathematics, 2000, vol. 71.

[34] M. Lazar, W.P. H. Heemels, and A. R. Teel, "Lyapunov functions, stability and input-to-state stability subtleties for discrete-time discontinuous systems," IEEE Trans. Autom. Control, vol. 54, no. 10, pp. 2421-2425, 2009.

[35] R. O. Duda and P. E. Hart, "Use of the hough transformation to detect lines and curves in pictures," Commun. ACM, vol. 15, no. 1, pp. 11-15, 1972.

[36] L. Hetel, C. Fiter, H. Omran, A. Seuret, E. Fridman, J.-P. Richard, and S. I. Niculescu, "Recent developments on the stability of systems with aperiodic sampling: An overview," Automatica, vol. 76, pp. 309-335, 2017.

[37] D. Du, W. Li, B. Zhan, M. Fei, and T. Yang, "Experimental performance analysis of inverted pendulum platform," in Theory, Methodology, Tools and Applications for Modeling and Simulation of Complex Systems. Singapore: Springer Singapore, 2016, pp. 431-440.

[38] K. Bansal and P. Mukhija, "Aperiodic sampled-data control of distributed networked control systems under stochastic cyber-attacks," IEEE/CAA J. Autom. Sinca, vol. 7, no. 4, pp. 1064-1073, 2020.

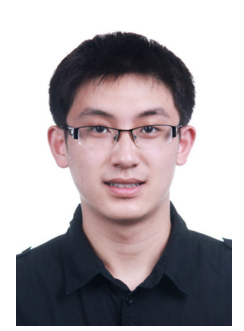

Yang Deng received the B.Sc. and M.Sc. degrees in information and computational science (belongs to applied mathematics, B.Sc. degree) and control engineering (M.Sc. degree) from Beihang University, Beijing, China, in 2014 and 2017, respectively. In 2020, he received the Ph.D. degree in automatic control engineering from École Centrale de Nantes, Nantes, France. Since 2021, he joined the Department of Automation, Tsinghua University, Beijing, China as a postdoctoral research associate. His research interests include the theory and application of time-delay systems, networked control systems, and robotic systems.

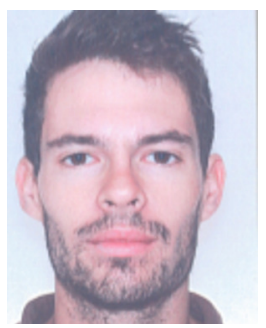

Vincent Lécheppé graduated in 2012 from the École Centrale de Nantes with a M.Sc. Degree in Automatic control. In 2015, he received his Ph.D. degree at the IRCCyN Laboratory in the École Centrale de Nantes. He was then a Lecturer in control system engineering at the University of South Pacific and a Post-doctoral Researcher Fellow at Griffith University, Australia. He is now an Assistant Professor at INSA Lyon France and Ampère Lab. His research interests include electrical machines control, predictor-based control, time-delay systems 


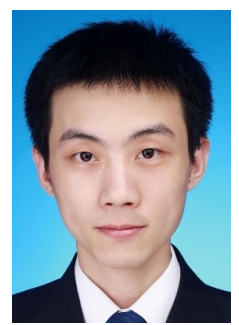

Changda Zhang received the B.Sc. and M.Sc. degrees from Shanghai University, Shanghai, China in 2016 and 2019, respectively. He is currently working toward the Ph. D. degree at Shanghai University. His main research interests include security control for networked control systems.

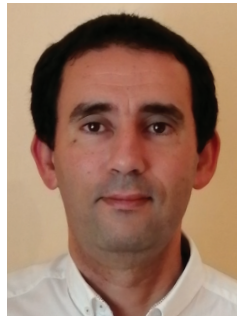

Emmanuel Moulay received his M.Sc. degree in mathematics from the University of Lille, France, in 2002. He obtained his Ph.D. degree in automatic control in 2005 from the École Centrale de Lille and his habilitation from the University of Poitiers in 2014. He joined the CNRS as a research scientist at the École Centrale de Nantes in 2006 and moved at the XLIM institute of the University of Poitiers in 2009. He is also adjunct professor in the department of Electrical and Computer Engineering at the University of Nebraska-Lincoln since 2020. His main interests are in control theory and its practical applications.

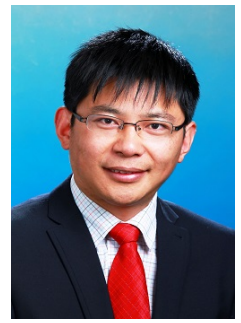

Dajun Du received the B.Sc. and M.Sc. degrees all from the Zhengzhou University, China in 2002 and 2005, respectively, and his Ph. D. degree in control theory and control engineering from Shanghai University in 2010. From September 2008 to September 2009, he was a visiting PHD student at Queen's University Belfast, UK. From April 2011 to August 2012, he was a Research Fellow at Queen's University Belfast, UK. He is currently a professor in Shanghai University. His main research interests include system identification and networked control

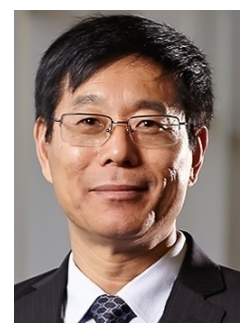

Qing-Long Han (M'09-SM'13-F'19) received the B.Sc. degree in Mathematics from Shandong Normal University, Jinan, China, in 1983, and the M.Sc. and $\mathrm{Ph} . \mathrm{D}$. degrees in Control Engineering from East China University of Science and Technology, Shanghai, China, in 1992 and 1997, respectively.

Professor Han is Pro Vice-Chancellor (Research Quality) and a Distinguished Professor at Swinburne University of Technology, Melbourne, Australia. He held various academic and management positions at Griffith University and Central Queensland University, Australia. His research interests include networked control systems, multi-agent systems, time-delay systems, smart grids, unmanned surface vehicles, and neural networks.

Professor Han was a Highly Cited Researcher in both Engineering and Computer Science (Clarivate Analytics, 2019-2020). He was one of Australia's Top 5 Lifetime Achievers (Research Superstars) in Engineering and Computer Science (The Australian's Research Magazine, 2019-2020). He was the recipient of The 2021 M. A. Sargent Medal (the Highest Award of the Electrical College Board of Engineers Australia), The 2020 IEEE Systems, Man, and Cybernetics (SMC) Society Andrew P. Sage Best Transactions Paper Award, The 2020 IEEE Transactions on Industrial Informatics Outstanding Paper Award, and The 2019 IEEE SMC Society Andrew P. Sage Best Transactions Paper Award.

Professor Han is a Member of the Academia Europaea (The Academy of Europe) and a Fellow of The Institution of Engineers Australia. He has served as an AdCom Member of IEEE Industrial Electronics Society (IES), a Member of IEEE IES Fellow Committee, and Chair of IEEE IES Technical Committee on Networked Control Systems. He is Co-Editor of Australian Journal of Electrical and Electronic Engineering, an Associate Editor for 12 international journals, including the IEEE TRANSACTIONS ON CYBERNETICS, the IEEE TRANSACTIONS ON INDUSTRIAL INFORMATICS, IEEE INDUSTRIAL ELECTRONICS MAGAZINE, the IEEE/CAA JOURNAL OF AUTOMATICA SINICA, Control Engineering Practice, and Information Sciences and Computing, and a Guest Editor for 13 Special Issues.

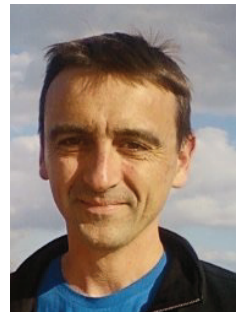

Franck Plestan (M'99) received the Ph.D. in $\mathrm{Au}-$ tomatic Control from the École Centrale de Nantes, France, in 1995. From September 1996 to August 2000, he was with Louis Pasteur University, Strasbourg, France. In September 2000, he joined the École Centrale de Nantes, Nantes, France where he is currently Professor. His research interests include robust control (adaptive/higher order sliding mode, time-delay systems) and nonlinear observer design. $\mathrm{He}$ is also working in several application domains as pneumatic actuators, automotive, flying systems, 